\title{
From coherent to incoherent mismatched interfaces: a generalized continuum formulation of surface stresses
}

\author{
Rémi Dingreville $^{a, 1}$, Abdelmalek Hallil $^{b}$ and Stéphane Berbenni ${ }^{c}$ \\ ${ }^{a}$ Sandia National Laboratories, Albuquerque, NM 87185, USA \\ ${ }^{b}$ Laboratoire des Sciences de l'Ingénieur pour l'Environnement (LaSIE), Université de La \\ Rochelle, Avenue Michel Crépeau, 17042 La Rochelle Cedex 1, France \\ ${ }^{c}$ Laboratoire d'Étude des Microstructures et de Mécanique des Matériaux, LEM3, UMR CNRS \\ 7239, Université de Lorraine, Ile du Saulcy, 57045 Metz, France
}

\section{Abstract}

The equilibrium of coherent and incoherent mismatched interfaces is reformulated in the context of continuum mechanics based on the Gibbs dividing surface concept. Two surface stresses are introduced: a coherent surface stress and an incoherent surface stress, as well as a transverse excess strain. The coherent surface stress and the transverse excess strain represent the thermodynamic driving forces of stretching the interface while the incoherent surface stress represents the driving force of stretching one crystal while holding the other fixed and thereby altering the structure of the interface. These three quantities fully characterize the elastic behavior of coherent and incoherent interfaces as a function of the in-plane strain, the transverse stress and the mismatch strain. The isotropic case is developed in detail and particular attention is paid to the case of interfacial thermo-elasticity. This exercise provides an insight on the physical significance of the interfacial elastic constants introduced in the formulation and illustrates the obvious coupling between the interface structure and its associated thermodynamics quantities. Finally, an example based on

\footnotetext{
${ }^{1}$ E-mail: rdingre@sandia.gov
}

Preprint submitted to Journal of the Mechanics and Physics of Solids

May 22, 2014

(C) 2014. This manuscript version is made available under the Elsevier user license http://www.elsevier.com/open-access/userlicense/1.0/ 
atomistic simulations of $\mathrm{Cu} / \mathrm{Cu}_{2} \mathrm{O}$ interfaces is given to demonstrate the relevance of the generalized interfacial formulation and to emphasize the dependence of the interfacial thermodynamic quantities on the incoherency strain with an actual material system.

Keywords: Surface stresses, interface properties, mismatch, grain boundaries.

1

2

3

5

\section{Introduction}

The physical and chemical properties of materials are greatly influenced by the presence of surrounding surfaces and interfaces, whether interfaces are in the form of grain boundaries separating two grains or in the case of a bicrystal between two dissimilar materials (phase boundaries). Interfacial stress and interfacial energy are quantities that play key roles in the thermodynamics of solid surfaces by providing a continuum description of the underlying atomistic complexity of interfaces. Through various constitutive interfacial models, the thermodynamics of interfaces has proven to be an important factor governing the behavior of materials systems separated by an interface or for which the surface to volume ratio is significant (Povstenko, 1993; Cammarata, 1994; Nix and Gao, 1998; Miller and Shenoy, 2000; Sharma et al., 2003; Dingreville et al., 2005; Duan et al., 2005; Chen et al., 2007; Duan and Karihaloo, 2007).

Indeed, atoms near an interface experience a different local environment than atoms in the bulk of a material. In the direction normal to a surface or an interface, the periodicity of the atomic lattice loses its translational symmetry over several atomic layers as demonstrated for example by atomistic simulations (Dingreville and $\mathrm{Qu}, 2007,2009$; Mi et al., 2008). In the case of an interface between two dissimilar materials, at least one phase must be elastically stretched due to the lattice mismatch between both phases resulting in the formation of defects such as dislocations or 
vacancies. Similarly, as a consequence, the equilibrium position and energy of atoms located in the vicinity of an interface are, in general, somewhat different from their bulk counterparts. Both the translational and in-plane atomic shuffling near the interface contribute to a change in the total energy of the interfacial system. The excess energy associated with atoms near an interface is called interfacial excess energy.

For incoherent interfaces (e.g those between two adjacent phases), relaxation of lattice mismatches may result in the formation of peculiar interfacial structures furthering the "excess thermodynamic state" over the bulk configuration. The state of interfacial coherency depends on the physical and chemical nature between both phases but is also inherently dependent on external factors such as the temperature or stress field. Qualitatively, the contacting phases possess mismatching lattice constants. For a coherent interface, the mismatch is completely accommodated by straining both phases. In the case of a semi-coherent interface, localized misfit dislocations are assumed to be responsible for compensating uniform far-field elastic fields, while an incoherent interface is the result of two rigid semi-infinite media in rigid contact (Romanov et al., 1998; Romanov and Wagner, 2001).

There are commonly three approaches adopted to study the properties of interfaces: (i) the diffuse interface model, (ii) the interphase model and (iii) the dividing interface/sharp interface model. In the case of the diffuse interface, interfacial properties are described by a smooth, but rapid transition of both fields (e.g., concentration or elastic fields) describing the interfacial properties (Leo et al., 1998; Hu and Chen, 2002). The interphase approach treats the interface in a classical thermodynamic fashion, namely, an arbitrarily defined finite volume is attributed to the interphase region (hence treated as a three-dimensional material system) and is assigned thermodynamic properties in a classical manner (Lipinski et al., 2006; Duan et al., 2007; 
Berbenni and Cherkaoui, 2010). Finally, the concepts of dividing surface, interfacial excess energy and interfacial stress are not new and were initially introduced by Gibbs (1928). In this paradigm, the surface contributions to the thermodynamic quantities are defined as the excesses over the values that would be obtained if the bulk phases retained their properties constant up to a two-dimensional imaginary dividing surface embedded in a three-dimensional continuum. In other words, the interface (not interphase) is a mathematical surface of zero thickness over which the thermodynamic properties change discontinuously from one bulk phase to the other. The excess amount is associated only with the dividing surface. This Gibbsian conceptual approach has been widely studied in the fields of physics and chemistry (Shuttleworth, 1950; Herring, 1951; Vermaak et al., 1968; Gurtin and Murdoch, 1975; Cahn and Larché, 1982; Nozières and Wolf, 1988; Cammarata and Sieradzki, 1994; Cammarata, 1994, 1997; Steigmann and Ogden, 1997; Gurtin et al., 1998; Nix and Gao, 1998; Cammarata et al., 2000; Sander, 2003; Müller and Saúl, 2004; Dingreville and Qu, 2008; Marichev, 2011; Mi and Kouris, 2012). Despite minor differences in the formulations, all interfacial models consist of the definition of an interfacial strain and the definition of an interfacial constitutive behavior linking the interfacial strain to the interface excess stress (also commonly called "surface stress"). Apart from a few exceptions, theories based on such two-dimensional framework cannot account for the flexural stiffness (Nozières and Wolf, 1988; Steigmann and Ogden, 1997, 1999; Gao et al., 2014) nor can they describe the transverse behavior of real material interfaces or account for the interfacial mismatch. Fairly recently, for a coherent interface in an elastic solid, the so-called Shuttleworth or ShuttleworthHerring relation (Shuttleworth, 1950; Herring, 1951) relating the interfacial excess energy $\Gamma$ to the surface stress $\Sigma^{S}$ has been generalized by Dingreville and Qu (2008) to account for the three-dimensional nature of the interface in a Gibbsian context 
such that,

$$
\boldsymbol{\Sigma}^{S}=\left.\frac{\partial \Gamma}{\partial \boldsymbol{\epsilon}^{S}}\right|_{\boldsymbol{\sigma}^{\perp}}+\boldsymbol{\sigma}^{\perp} \cdot \underline{\mathbb{H}},
$$

where the surface stress $\boldsymbol{\Sigma}^{S}$ is not only a function of the interface in-plane strain $\boldsymbol{\epsilon}^{S}$ (which is the case in the original Shuttleworth relation) but also a function of the transverse stress $\boldsymbol{\sigma}^{\perp}$. The third-order interfacial tensor $\underline{\mathbb{H}}$ measures the inherent Poisson's effect in the transverse direction of the interface. However, in the case of a semi-coherent or incoherent interface, as discussed by Cahn and Larché (1982), it is necessary to define two strain and stress measures to describe the general deformation at the interface, rendering equation (1) inadequate since both phases can in principle be stretched independently along the interface.

The present work proposes the use of the Gibbs dividing surface thermodynamic framework to develop generalized expressions for interfacial excess stresses and interfacial excess energy and to account for their variations with respect to the in-plane surface strain, the transverse stress and the interface mismatch strain. Our goal is to unambiguously define interface stresses and the interfacial excess strain in order to understand the connection between the interface thermodynamic quantities and the interface kinetic quantities to arrive at a generalized Shuttleworth relation for mismatched interfaces. In essence, our approach is close to that of Cahn and Larché (1982); in fact, we mostly render their formulation more general and systematic.

The manuscript is organized as follows. Section 2 starts with the definitions of the interfacial kinematics. In sections 3 and 4 , we propose a generalized formulation of the Shuttleworth relationship when a structural mismatch is present at the interface. Explicit expressions of the interfacial tensors are derived in the special case of an incoherent interface between two dissimilar isotropic solids. The connection between the structural mismatch and elastic properties of interfaces is revealed 
in the singular case of isotropic thermo-elasticity. Finally, numerical examples are provided in section 5 for incoherent interfaces between $\mathrm{Cu}$ and its oxide $\mathrm{Cu}_{2} \mathrm{O}$ to illustrate the physicality and insight provided by the proposed formulation in view of atomistic simulations. Note that in the following sections of this manuscript, the terms interface and surface are used interchangeably. A similar remark applies to the designation of the Shuttleworth or Shuttleworth-Herring relationship.

A list of notation and convention used throughout this manuscript is cataloged in Appendix A.

\section{Interfacial kinematics}

Let us consider an incoherent planar interface between two dissimilar elastic crystalline phases subjected to an anisotropic and nonuniform elastic deformation. The two half spaces are assumed to be mutually insoluble so that no diffusion occurs between them and can be ignored. In the interest of a concise analysis, we choose to consider only processes and equilibria at constant and uniform temperature (hence neglecting configurational and thermal entropies). Therefore, and although an explicit temperature dependence is admitted for all free energy functions and materials parameters, we do not display the temperature as a variable (lattice constants and materials properties may be a function of the temperature). It is also assumed that, if more than one interface is present in the the material system, these interfaces do not interact with one another. For thin slabs and small particles, it has been demonstrated that interfaces may interact with one another, altering interfacial properties and showing a finite size effect (Zhang et al., 2014). This size effect is more pronounced for ionic systems (long range electrostatic interaction forces) than it is for more classical metallic systems. 
We begin with the definitions of interfacial strains and incoherency. The interface $\mathcal{S}$ is considered as an imaginary, two-dimensional surface with its location chosen arbitrarily following the Gibbs dividing surface concept. For convenience, let the interface be located at $z=0$ in a Cartesian coordinate system as shown in Fig 1 . In this work we consider that the bicrystal is subjected to a homogeneous traction and displacement boundary condition at infinity, such that far away from the interface the deformation can be assumed homogeneous, and the transverse (second PiolaKirchhoff) stress and the in-plane (Lagrangian) strain tensors in the upper $(z>0)$ and lower $(z<0)$ materials are given by $\boldsymbol{\sigma}_{ \pm}^{\perp}$ and $\boldsymbol{\epsilon}_{ \pm}^{S}$ respectively. It has been shown that such homogeneous deformation can be easily constructed in a bimaterial $(\mathrm{Qu}$ and Bassani, 1993) by the so-called "T-decomposition". Although the deformation far away from the interface is homogeneous, the stress and strain fields near the interface are disrupted by the presence of the interface and the associated disturbance of the atomic lattice periodicity in its vicinity. The deformation of the interfacial system manifests itself by displacements of the atoms $i$ which can be described by individual displacement vectors $\mathbf{v}^{(i)}$. In general, the deformation field gives rise to local stresses in the interfacial system, which can be related by balance laws to the stress in the interface surroundings and external forces acting on the atoms near the interface. If we assume that the displacement varies smoothly at the atomic scale, the equations of balance can be derived based on a description of the interfacial system as a continuum, where the displacement field is represented by $\mathbf{u}(\mathbf{x})$, with the vector $\mathbf{x}$ being a continuous position variable. The treatment of the displacement fields and strain fields is measured from a reference state (Lagrangian measure) of one of the two half crystals chosen arbitrarily. The reference states for medium "+" $(z>0)$ and for medium "-_ $(z<0)$ can be chosen as states of uniform composition. As mentioned by Cahn and Larché (1982), two measures of the Lagrangian in- 
terfacial in-plane strain tensors, $\boldsymbol{\epsilon}^{S}$ and $\boldsymbol{\epsilon}^{*, S}$ respectively, are necessary since each crystal has its own reference state. The displacement fields $\mathbf{u}_{+}(\mathbf{x})$ and $\mathbf{u}_{-}(\mathbf{x})$ can be used for the standard definition of the elastic in-plane surface strain tensor in small deformation $\boldsymbol{\epsilon}_{+}^{S}$ and $\boldsymbol{\epsilon}_{-}^{S}$ in medium "+" and medium "-" respectively such that,

$$
\boldsymbol{\epsilon}_{ \pm}^{S}=\frac{1}{2}\left(\nabla^{S} \mathbf{u}_{ \pm}+\nabla^{S} \mathbf{u}_{ \pm}^{T}\right)
$$

where $\nabla^{S}$ is the surface gradient. The surface nabla operator $\nabla^{S}$ is simply defined by the projection of the nabla operator $\nabla$ on the tangential plane of the interface such that,

$$
\nabla^{S}=\left(\mathbf{I}-\mathbf{n}\left(\mathbf{x}^{S}\right) \otimes \mathbf{n}\left(\mathbf{x}^{S}\right)\right) \nabla
$$

where $\mathbf{n}\left(\mathbf{x}^{S}\right)$ is the interface unit normal upward pointing from the reference phase (in this case the "+" half space), $\mathbf{x}^{S}$ is the interfacial position vector, and $\mathbf{I}$ is the second-order identity tensor. Having the medium "+" chosen as our reference phase and assuming continuity of displacement along the interface leads to $\boldsymbol{\epsilon}^{S}=\boldsymbol{\epsilon}_{+}^{S}=\boldsymbol{\epsilon}_{-}^{S}$. Because medium " + " is chosen as our reference, $\boldsymbol{\epsilon}^{S}$ is independent of $\mathbf{u}_{-}(\mathbf{x})$. The in-plane strain tensor $\epsilon^{S}$ represents the interface strain measure associated with deforming both phases by the same amount. Another assumption stemming for the "T- decomposition" (see Appendix B for more details) leads to an interfacial normal stress continuity condition (i.e. $\boldsymbol{\sigma}^{\perp}=\boldsymbol{\sigma}_{+}^{\perp}=\boldsymbol{\sigma}_{-}^{\perp}$ ).

The second strain measure $\boldsymbol{\epsilon}^{*, S}$ associated with the interface represents the interface components of the strain of medium "-" relative to medium "+". As illustrated in Fig. 2, following Romanov and Wagner (2001) and Cahn and Larché (1982), the strain tensor $\boldsymbol{\epsilon}^{*, S}$ is only defined in medium "-" such that it can be expressed and decomposed into two components such that,

$$
\boldsymbol{\epsilon}^{*, S}(\mathbf{x})=\boldsymbol{\epsilon}_{-}^{*, S}(\mathbf{x})=\frac{1}{2}\left[\left(\nabla^{S} \mathbf{u}_{-}-\nabla^{S} \mathbf{u}_{+}\right)+\left(\nabla^{S} \mathbf{u}_{-}-\nabla^{S} \mathbf{u}_{+}\right)^{\mathrm{T}}\right]=\boldsymbol{\epsilon}^{0, S}+\boldsymbol{\epsilon}^{m, S} g(\mathbf{x}) .
$$


The strain tensor $\boldsymbol{\epsilon}^{0, S}$ is an eigenstrain related to stress free configuration corresponding to the change in molar volume between medium "+" and medium "-". For cubic systems, the molar volume $V_{-}^{0}$ of stress free medium "-" is given in terms of the molar volume $V_{+}^{0}$ of medium "+" in its reference state such that,

$$
V_{-}^{0}=\left[1+\epsilon_{k k}^{0, S}\right]^{3} V_{+}^{0} \approx\left[1+3 \epsilon_{k k}^{0, S}\right] V_{+}^{0} .
$$

The strain tensor $\boldsymbol{\epsilon}^{m, S}$ represents the in-plane structural mismatch or deviation from the coherency at the interface (i.e. $z=0$ ). The spatial function $g(\mathbf{x})$ defined in equation (4) corresponds to the variation of this structural mismatch over few atomic layers and vanishes far from the interface. As such, $g(0)=1$ by definition of the in-plane structural mismatch $\boldsymbol{\epsilon}^{m, S}$, and $g(\mathbf{x})=0, \forall \mathbf{x} \leq-h$, where $h$ is an arbitrary distance far away from the interface (see illustrations in Fig. 1 and Fig. 2). In the present manuscript (semi-infinite media), the function $g(\mathbf{x})$ does not vary with the interfacial material system size (as it would be the case for thin films, for example) since we are not accounting for interfaces interactions. The structural mismatch at the interface is usually defined as (Romanov et al., 1998; Sayle et al., 2002):

$$
\epsilon_{\alpha \beta}^{m, S}=2 \frac{\ell_{+}-\ell_{-}}{\ell_{+}+\ell_{-}} \delta_{\alpha \beta},
$$

where $\ell_{+}$and $\ell_{-}$are the crystal lattice parameters in phase "+" and "-" respectively and $\delta_{\alpha \beta}$ is the Kronecker delta symbol.

In light of the definitions of $\boldsymbol{\epsilon}^{0, S}$ and $\boldsymbol{\epsilon}^{m, S}$, the strain tensor $\boldsymbol{\epsilon}^{*, S}$ corresponds to an in-plane eigenstrain related to the change of the interface structure. For example, if we consider the interface between a crystal of finite thickness and a flat thick substrate, changing the in-plane lattice spacing of the crystal while keeping the lattice spacing of the substrate constant requires the introduction of misfit dislocations at the interface to relax the interfacial system (Romanov et al., 1998). 


\section{Thermodynamic framework for an incoherent interface}

\subsection{Interfacial thermodynamic framework}

We now use the Gibbs dividing surface thermodynamic framework to define the interfacial excess free energy, interfacial excess stress and interfacial excess strain for a coherent and incoherent interface. In this continuum representation, the total free energy $\mathcal{F}$ of the interfacial system (i.e. both semi-infinite crystal $\Omega_{+}$and $\Omega_{-}$and the dividing interface $\mathcal{S}$ ) is given by

$$
\mathcal{F}=\int_{\Omega_{+}} \Psi_{+}(\mathbf{x}) d V+\int_{\Omega_{-}} \Psi_{-}(\mathbf{x}) d V+\int_{\mathcal{S}} \Gamma(\mathbf{x}) d A
$$

where $\Psi_{ \pm}(\mathbf{x})$ is the bulk free-energy density per unit volume in medium "+" and "-" respectively, while $\Gamma(\mathbf{x})$ is the interfacial excess free energy.

Both bulk phases are being strained by the same amount in the plane of the interface and loaded transversely to the interface along its normal direction ("Tdecomposition"). Additionally each solid half space is subjected to eigenstrains $\boldsymbol{\epsilon}_{ \pm}^{*}(\mathbf{x})\left(\boldsymbol{\epsilon}_{+}^{*}(\mathbf{x})=0\right)$ in medium "+" or medium "-" respectively and the bulk constitutive behavior is treated in a classical manner such that,

$$
\begin{aligned}
\Psi_{ \pm} & =\hat{\Psi}_{ \pm}\left(\nabla \mathbf{u}, \boldsymbol{\epsilon}^{*}, \mathbf{x}\right) \text { in } \Omega_{ \pm} \\
d \Psi_{ \pm} & =\boldsymbol{\sigma}_{ \pm}: d \boldsymbol{\epsilon}_{ \pm}=\underline{\underline{C}}_{ \pm}:\left(\boldsymbol{\epsilon}_{ \pm}-\boldsymbol{\epsilon}_{ \pm}^{*}\right) d \boldsymbol{\epsilon}_{ \pm}
\end{aligned}
$$

where $\boldsymbol{\sigma}_{ \pm}$is the second Piola-Kirchhoff stress tensor in medium "+" or medium "-" respectively, $\underline{\underline{C}}_{ \pm}$is the corresponding elastic stiffness tensor and the elastic strain tensor in small deformation $\boldsymbol{\epsilon}_{ \pm}$is defined in a standard fashion. The bulk description is augmented by the constitutive behavior of the interface in the form,

$$
\Gamma=\hat{\Gamma}\left(\boldsymbol{\epsilon}^{S}, \boldsymbol{\epsilon}^{m, S}, \boldsymbol{\sigma}^{\perp}, \mathbf{x}^{S}\right)
$$


where $\boldsymbol{\epsilon}^{S}$ is the surface strain defined in equation (2), $\boldsymbol{\sigma}^{\perp}$ denotes the transverse stress tensor normal to the interface (based on the "T-decomposition") and $\boldsymbol{\epsilon}^{m, S}$ is the in-plane structural mismatch strain tensor defined in equation (4).

The variables $\left(\boldsymbol{\epsilon}^{S}, \boldsymbol{\sigma}^{\perp}, \boldsymbol{\epsilon}^{m, S}\right)$ being defined, the next task is to identify their appropriate interfacial conjugate variables through the generalized thermodynamic framework for the interface free energy density for which the interfacial power density of internal forces takes the form in the most general case,

$$
p^{S}=\boldsymbol{\Sigma}^{S}: \dot{\boldsymbol{\epsilon}}^{S}+\mathbb{D}^{\perp} \cdot \dot{\boldsymbol{\sigma}}^{\perp}+\Upsilon^{S}: \dot{\boldsymbol{\epsilon}}^{m, S}
$$

In this context, the surface tensor $\Sigma^{S}$ can be identified as the interfacial stress, thermodynamic conjugate of the in-plane strain field $\boldsymbol{\epsilon}^{S}$, the surface tensor $\Upsilon^{S}$ can be identified as the incoherent interfacial stress, thermodynamic conjugate of the interfacial mismatch strain $\epsilon^{m, S}$, and the tensor $\mathbb{D}^{\perp}$ can be associated with the interfacial excess strain, thermodynamic conjugate of the transverse stress field $\boldsymbol{\sigma}^{\perp}$. For coherent interfaces the last term is missing since $\boldsymbol{\epsilon}^{m, S}=0$. The term in $\Upsilon^{S}: \boldsymbol{\epsilon}^{m, S}$ can be viewed as a correction to the interfacial energy due to deviation from coherency. Expressions for tensor $\Sigma^{S}, \mathbb{D}^{\perp}$ and $\Upsilon^{S}$ stated as a function of the interfacial excess energy and its derivatives with respect to the state variables $\left(\boldsymbol{\epsilon}^{S}, \boldsymbol{\sigma}^{\perp}, \boldsymbol{\epsilon}^{m, S}\right)$ constitute the generalized Shuttleworth relationship (see section 3.5).

\subsection{Interfacial excess energy}

The interfacial excess energy $\Gamma$ of a mismatched interface is defined using the standard Gibbs definition of the interfacial excess energy per unit of undeformed area combined with the "T-decomposition" (see Appendix B) such that,

$$
\Gamma=\int_{0}^{\infty}\left(\Psi(\mathbf{x})-\Psi_{+}\right) d \mathbf{x}+\int_{-\infty}^{0}\left(\Psi(\mathbf{x})-\Psi_{-}\right) d \mathbf{x}
$$


where

$$
\begin{aligned}
\Psi(\mathbf{x}) & =\hat{\Psi}_{0}+\hat{\boldsymbol{\tau}}^{S}(\mathbf{x}):\left[\boldsymbol{\epsilon}^{S}-\boldsymbol{\epsilon}^{*, S}(\mathbf{x})\right] \\
& +\frac{1}{2}\left[\boldsymbol{\epsilon}^{S}-\boldsymbol{\epsilon}^{*, S}(\mathbf{x})\right]: \underline{\mathbb{C}}^{S}(\mathbf{x}):\left[\boldsymbol{\epsilon}^{S}-\boldsymbol{\epsilon}^{*, S}(\mathbf{x})\right]+\frac{1}{2} \boldsymbol{\sigma}^{\perp} \cdot \mathbb{M}^{\perp}(\mathbf{x}) \cdot \boldsymbol{\sigma}^{\perp}
\end{aligned}
$$

230

and

$$
\begin{aligned}
& \Psi_{+}=\frac{1}{2} \boldsymbol{\epsilon}^{S}: \underline{\underline{\mathbb{C}}}_{+}^{S}: \boldsymbol{\epsilon}^{S}+\frac{1}{2} \boldsymbol{\sigma}_{+}^{\perp} \cdot \mathbb{M}_{+}^{\perp} \cdot \boldsymbol{\sigma}_{+}^{\perp}, \\
& \Psi_{-}=\frac{1}{2}\left(\boldsymbol{\epsilon}^{S}-\boldsymbol{\epsilon}^{0, S}\right): \underline{\underline{\mathbb{C}}}_{-}^{S}:\left(\boldsymbol{\epsilon}^{S}-\boldsymbol{\epsilon}^{0, S}\right)+\frac{1}{2} \boldsymbol{\sigma}^{\perp} \cdot \mathbb{M}_{-}^{\perp} \cdot \boldsymbol{\sigma}^{\perp} .
\end{aligned}
$$

231

232

233 234

Keep in mind that equations (14) and (15) are the result of having medium "+" as the reference medium. As such, the eigenstrain $\boldsymbol{\epsilon}^{0, S}$ appears only in medium "_" and not in medium "+". Making use of equations (13), (14) and (15) in equation (12), it follows that the interfacial excess energy $\Gamma$ can be expressed as,

$$
\begin{aligned}
\Gamma & =\Gamma_{0}+\boldsymbol{\Gamma}^{(1)}: \boldsymbol{\epsilon}^{S}+\frac{1}{2} \boldsymbol{\epsilon}^{S}: \underline{\underline{\Gamma}}^{(2)}: \boldsymbol{\epsilon}^{S}+\frac{1}{2} \boldsymbol{\sigma}^{\perp} \cdot \boldsymbol{\Lambda}^{(2)} \cdot \boldsymbol{\sigma}^{\perp} \\
& +\boldsymbol{\Upsilon}^{(1)}: \boldsymbol{\epsilon}^{m, S}+\frac{1}{2} \boldsymbol{\epsilon}^{m, S}: \underline{\underline{\Upsilon}}^{(2)}: \boldsymbol{\epsilon}^{m, S}-\boldsymbol{\epsilon}^{m, S}: \underline{\underline{\boldsymbol{\Phi}}}: \boldsymbol{\epsilon}^{S}
\end{aligned}
$$


where,

$$
\begin{aligned}
& \Gamma_{0}=\int_{-\infty}^{\infty} \hat{\Psi}_{0}(\mathbf{x}) d \mathbf{x}-\int_{0}^{\infty} \hat{\boldsymbol{\tau}}^{S}(\mathbf{x}) \epsilon^{0, S} d \mathbf{x} \\
& +\frac{1}{2} \int_{-\infty}^{0} \boldsymbol{\epsilon}^{0, S}:\left[\underline{\underline{C}}^{S}(\mathbf{x})-\underline{\underline{C}}_{-}^{S}\right]: \boldsymbol{\epsilon}^{0, S} d \mathbf{x} \\
& \boldsymbol{\Gamma}^{(1)}=\int_{-\infty}^{\infty} \hat{\boldsymbol{\tau}}^{S}(\mathbf{x}) d \mathbf{x}-\int_{-\infty}^{0} \boldsymbol{\epsilon}^{0, S}:\left[\underline{\underline{\mathbb{C}}}^{S}(\mathbf{x})-\underline{\underline{\mathbb{C}}}_{-}^{S}\right] d \mathbf{x}, \\
& \underline{\underline{\Gamma}}^{(2)}=\int_{0}^{\infty}\left[\underline{\underline{\mathbb{C}}}^{S}(\mathbf{x})-\underline{\underline{\mathbb{C}}}_{+}^{S}\right] d \mathbf{x}+\int_{-\infty}^{0}\left[\underline{\underline{\mathbb{C}}}^{S}(\mathbf{x})-\underline{\mathbb{C}}_{-}^{S}\right] d \mathbf{x} \\
& \boldsymbol{\Upsilon}^{(1)}=-\int_{-\infty}^{0} \hat{\boldsymbol{\tau}}^{S}(\mathbf{x}) g(\mathbf{x}) d \mathbf{x}+\int_{-\infty}^{0} \boldsymbol{\epsilon}^{0, S}: \underline{\underline{C}}^{S}(\mathbf{x}) g(\mathbf{x}) d \mathbf{x}, \\
& \underline{\underline{\Upsilon}}^{(2)}=\int_{-\infty}^{0} \underline{\underline{\mathbb{C}}}^{S}(\mathbf{x}) g^{2}(\mathbf{x}) d \mathbf{x} \\
& \Lambda^{(2)}=\int_{0}^{\infty}\left[\mathbb{M}^{\perp}(\mathbf{x})-\mathbb{M}_{+}^{\perp}\right] d \mathbf{x}+\int_{0}^{\infty}\left[\mathbb{M}^{\perp}(\mathbf{x})-\mathbb{M}_{-}^{\perp}\right] d \mathbf{x} \\
& \underline{\underline{\Phi}}=\int_{-\infty}^{0} \underline{\mathbb{C}}^{S}(\mathbf{x}) g(\mathbf{x}) d \mathbf{x} \text {. }
\end{aligned}
$$

Note that, even though the range of the integrals defined in equations (17)-(23) is infinite, the convergence of these integrals is satisfied. Indeed $\hat{\boldsymbol{\tau}}^{S}(\mathbf{x})$ and $g(\mathbf{x})$ both vanish far away from the interface so that the integrals only need to be carried out over a finite thickness in the interphase region (see Fig. 1(b) and Fig. 2). Equation (16) expresses the interfacial excess energy as a function of the in-plane strain $\boldsymbol{\epsilon}^{S}$, the transverse stress $\boldsymbol{\sigma}^{\perp}$ and the interfacial mismatch $\boldsymbol{\epsilon}^{m, S}$. As illustrated in equation (24), note the explicit dependence and correction of the residual interfacial excess energy $\Gamma_{0}$ and some of the interfacial elastic tensors due to the state of coherency of the interface through the eigenstrain $\epsilon^{0, S}$ and the short range function $g(\mathbf{x})$ :

$$
\left.\Gamma\right|_{\boldsymbol{\epsilon}^{S}=0, \boldsymbol{\sigma}^{\perp}=0}=\Gamma^{*}=\Gamma_{0}+\boldsymbol{\Upsilon}^{(1)}: \boldsymbol{\epsilon}^{m, S}+\frac{1}{2} \boldsymbol{\epsilon}^{m, S}: \underline{\underline{\Upsilon}}^{(2)}: \boldsymbol{\epsilon}^{m, S}
$$




\subsection{In-plane interfacial excess stress}

We now define the in-plane interfacial excess stress by decomposing the second Piola-Kirchhoff stress tensor in its in-plane component and transverse component (see equation (B.9) in Appendix B), so that we obtain

$$
\boldsymbol{\sigma}^{S}(\mathbf{x})=\hat{\boldsymbol{\tau}}^{S}(\mathbf{x})+\underline{\underline{\mathbb{C}}}^{S}(\mathbf{x}):\left[\boldsymbol{\epsilon}^{S}-\boldsymbol{\epsilon}^{*, S}(\mathbf{x})\right]+\boldsymbol{\sigma}^{\perp} \cdot \underline{\boldsymbol{\gamma}}(\mathbf{x})
$$

Furthermore in the bulk of medium "+" and medium "-",

$$
\begin{aligned}
& \boldsymbol{\sigma}_{+}^{S}=\underline{\mathbb{C}}_{+}^{S}: \boldsymbol{\epsilon}_{+}^{S}+\boldsymbol{\sigma}^{\perp} \cdot \underline{\gamma}_{+}, \\
& \boldsymbol{\sigma}_{-}^{S}=\underline{\underline{\mathbb{C}}}_{-}^{S}:\left(\boldsymbol{\epsilon}_{-}^{S}-\boldsymbol{\epsilon}^{0, S}\right)+\boldsymbol{\sigma}^{\perp} \cdot \underline{\boldsymbol{\gamma}}_{-} .
\end{aligned}
$$

Again equations (26) and (27) are the result of having medium "+" as the reference medium. Following Dingreville and Qu (2008), we can define the (second PiolaKirchhoff) interfacial excess stress by,

$$
\boldsymbol{\Sigma}^{S}=\int_{0}^{\infty}\left(\boldsymbol{\sigma}^{S}(\mathbf{x})-\boldsymbol{\sigma}_{+}^{S}\right) d \mathbf{x}+\int_{-\infty}^{0}\left(\boldsymbol{\sigma}^{S}(\mathbf{x})-\boldsymbol{\sigma}_{-}^{S}\right) d \mathbf{x} .
$$

It results from strain compatibility $\left(\boldsymbol{\epsilon}^{S}=\boldsymbol{\epsilon}_{+}^{S}=\boldsymbol{\epsilon}_{-}^{S}\right)$, traction continuity $\left(\boldsymbol{\sigma}^{\perp}=\boldsymbol{\sigma}_{+}^{\perp}=\right.$ $\left.\boldsymbol{\sigma}_{-}^{\perp}\right)$ and equations $(25)-(27)$ that,

$$
\boldsymbol{\Sigma}^{S}=\Gamma^{(1)}-\underline{\underline{\Phi}}: \boldsymbol{\epsilon}^{m, S}+\underline{\underline{\Gamma}}^{(2)}: \boldsymbol{\epsilon}^{S}+\boldsymbol{\sigma}^{\perp} \cdot \underline{\mathbb{H}},
$$

where $\Gamma^{(1)}, \underline{\underline{\Gamma}}^{(2)}, \underline{\underline{\Phi}}$ are given by equations (18)-(23) and $\underline{\mathbb{H}}$ reads

$$
\underline{\mathbb{H}}=\int_{0}^{\infty}\left[\underline{\boldsymbol{\gamma}}(\mathbf{x})-\underline{\boldsymbol{\gamma}}_{+}\right] d \mathbf{x}+\int_{-\infty}^{0}\left[\underline{\boldsymbol{\gamma}}(\mathbf{x})-\underline{\boldsymbol{\gamma}}_{-}\right] d \mathbf{x} .
$$

The first term $\Gamma^{(1)}$ represents the internal residual excess stress of the interface. This component of the surface stress exists even when a remote interfacial deformation is absent. Note that two factors contribute to this residual surface stress (see equation (18)). One can be attributed to the pure elastic behavior of the interface (see 
term in $\hat{\boldsymbol{\tau}}^{S}$ in equation (18)). This contribution has been discussed and derived in a similar fashion by others (Cammarata, 1994; Nix and Gao, 1998). The second contribution can be ascribed to the coupling between the state of coherency, the change in molar volume between the two media and the elastic stiffness of the interface (see term in $\boldsymbol{\epsilon}^{0, S}$ in equation (18)). The term $\underline{\boldsymbol{\Phi}}$ describes the intrinsic coupling between the surface stress and the structural interfacial mismatch. The third term $\underline{\underline{\Gamma}}^{(2)}$ represents the interface in-plane elastic stiffness tensor, while the tensor $\underline{\mathbb{H}}$ measures the Poisson's effect of the interface in the direction normal to the interface. Note that the interface coherency does not affect the surface stiffness (surface elastic tensors $\underline{\underline{\Gamma}}^{(2)}$ and $\underline{\mathbb{H}}$ ), but does have an effect on the residual surface stress $\Gamma^{(1)}$ and the coupling with the structural mismatch $\underline{\underline{\Phi}}$. As such the first two terms of equation (29) could be viewed as a correction to the coherent residual surface stress due to the mismatch at the interface, while the last two terms are solely due to the elastic stiffness and compliance of the interface.

\subsection{Transverse interfacial excess strain}

Similarly, if we consider the transverse interfacial excess strain by decomposing the transverse Lagrangian strain according to the "T-decomposition" (see equation (B.7) in Appendix B), we get,

$$
\boldsymbol{\epsilon}^{\perp}(\mathbf{x})=\epsilon^{\perp, *}(\mathbf{x})-\mathbb{M}^{\perp}(\mathbf{x}) \cdot \tau^{\perp}(\mathbf{x})+\mathbb{M}^{\perp}(\mathbf{x}) \cdot \sigma^{\perp}-\underline{\gamma}(\mathbf{x}):\left[\epsilon^{S}-\epsilon^{*, S}(\mathbf{x})\right] .
$$

with the transverse strain in the bulk of each medium given by,

$$
\begin{aligned}
& \boldsymbol{\epsilon}_{+}^{\perp}=\mathbb{M}_{+}^{\perp} \cdot \sigma^{\perp}-\underline{\gamma}_{+}: \boldsymbol{\epsilon}^{S}, \\
& \boldsymbol{\epsilon}_{-}^{\perp}=\boldsymbol{\epsilon}^{\perp, 0}+\mathbb{M}_{-}^{\perp} \cdot \boldsymbol{\sigma}^{\perp}-\underline{\gamma}_{-}:\left(\boldsymbol{\epsilon}^{S}-\boldsymbol{\epsilon}^{0, S}\right) .
\end{aligned}
$$

Here again equations (32) and (33) are the result of having medium "+" as the reference medium. 
The excess transverse interfacial strain is defined by,

$$
\boldsymbol{\Delta}^{\perp}=\int_{0}^{\infty}\left(\boldsymbol{\epsilon}^{\perp}(\mathbf{x})-\boldsymbol{\epsilon}_{+}^{\perp}\right) d \mathbf{x}+\int_{-\infty}^{0}\left(\boldsymbol{\epsilon}^{\perp}(\mathbf{x})-\boldsymbol{\epsilon}_{-}^{\perp}\right) d \mathbf{x}
$$

which becomes,

$$
\boldsymbol{\Delta}^{\perp}=\boldsymbol{\Lambda}^{(1)}+\underline{\mathbb{K}}: \boldsymbol{\epsilon}^{m, S}+\boldsymbol{\Lambda}^{(2)} \cdot \boldsymbol{\sigma}^{\perp}-\underline{\mathbb{H}}: \boldsymbol{\epsilon}^{S},
$$

with the tensors $\boldsymbol{\Lambda}^{(1)}$ and $\underline{\mathbb{K}}$ defined by,

$$
\begin{aligned}
\Lambda^{(1)} & =\int_{-\infty}^{0}\left(\boldsymbol{\epsilon}^{\perp, *}(\mathbf{x})-\boldsymbol{\epsilon}^{\perp, 0}\right)+(\underline{\boldsymbol{\gamma}}(\mathbf{x})-\underline{\boldsymbol{\gamma}}): \boldsymbol{\epsilon}^{0, S} d \mathbf{x} \\
& -\int_{-\infty}^{\infty} \mathbb{M}^{\perp}(\mathbf{x}) \cdot \boldsymbol{\tau}^{\perp}(\mathbf{x}) d \mathbf{x}, \\
\underline{\mathbb{K}} & =\int_{-\infty}^{0} \underline{\boldsymbol{\gamma}}(\mathbf{x}) g(\mathbf{x}) d \mathbf{x} .
\end{aligned}
$$

The transverse interfacial excess strain tensor $\boldsymbol{\Delta}^{\perp}$ measures the interfacial excess deformation in the direction transverse to the interface. The first term $\Lambda^{(1)}$ represents the part of transverse interfacial deformation that exists even when a remote interfacial deformation is absent. Its physical interpretation could be viewed as an "intrinsic relaxed thickness" of the interface as compared with an interface which would retain a perfect bulk lattice arrangement up to the dividing surface. Similarly to the residual surface stress, two elements compose the "intrinsic relaxed thickness" of the interface. One comes from the elastic transverse compliance across the interface (see term in $\mathbb{M}^{\perp}$ in equation (36)) and the other stems from the coupling between the interface elastic behavior and the interface state of coherency (see term in $\boldsymbol{\epsilon}^{0, S}$ in equation (36)). The tensor $\boldsymbol{\Lambda}^{(2)}$ describes the transverse compliance of the interface, while the tensor $\mathbb{K}$ represents the coupling between the transverse loading and the structural mismatch at the interface. Analogously to the remarks made for the in-plane interfacial excess stress, note that the first two terms of equation (35) could be viewed as a correction to the "intrinsic relaxed thickness" for a coherent 
interface due to the mismatch at the interface, while the surface coherency does not affect the interface elastic compliance (tensor $\boldsymbol{\Lambda}^{(2)}$ ).

\subsection{Generalized Shuttleworth relationship}

Based on the thermodynamic derivations presented above, the generalized form of the Shuttleworth relationship for a mismatched interface can be obtained. By combining equations (11) with equations (16) and (29), the relation between the coherent surface stress $\boldsymbol{\Sigma}^{S}$ and the interfacial energy $\Gamma$ is given by,

$$
\boldsymbol{\Sigma}^{S}=\left.\frac{\partial \Gamma}{\partial \boldsymbol{\epsilon}^{S}}\right|_{\boldsymbol{\epsilon}^{m, S}, \boldsymbol{\sigma}^{\perp}}+\boldsymbol{\sigma}^{\perp} \cdot \underline{\mathbb{H}},
$$

while the relation between the interfacial strain $\Delta^{\perp}$ and the interfacial energy $\Gamma$ is simply obtained by combining equations (11) with equations (16) and (35) such that,

$$
\mathbb{D}^{\perp}=\boldsymbol{\Delta}^{\perp}-\boldsymbol{\Lambda}^{(1)}=\left.\frac{\partial \Gamma}{\partial \boldsymbol{\sigma}^{\perp}}\right|_{\boldsymbol{\epsilon}^{S}, \boldsymbol{\epsilon}^{m, S}}-\underline{\mathbb{I}}: \boldsymbol{\epsilon}^{S}+\underline{\mathbb{K}}: \boldsymbol{\epsilon}^{m, S} .
$$

Note that equations (38) and (39) are consistent with that of Dingreville and Qu (2008) in the above equation (1) and reduce to the original formulation of ShuttleworthHerring when $\boldsymbol{\sigma}^{\perp}=0$ and $\boldsymbol{\epsilon}^{m, S}=0$. The term $\boldsymbol{\Lambda}^{(1)}+\underline{\mathbb{K}}: \boldsymbol{\epsilon}^{m, S}$ corresponds to a correction due to the structure of the interface (through the mismatch strain) to the intrinsic "relaxation width" of the interface accounting for the relative movement between the top and bottom "surfaces" of the interfacial region, even when $\boldsymbol{\sigma}^{\perp}=0$ and $\boldsymbol{\epsilon}^{S}=0$ (i.e., when the remote traction the in-plane strain vanishes). Additionally, the coherent residual surface stress comprises a correction due to (i) the change in molar volume between medium "+" and medium "-" through the eigenstrain $\epsilon^{0, S}$ in the two-dimensional tensor $\boldsymbol{\Gamma}^{(1)}$ and (ii) the intrinsic misfit structural coupling through the tensor $\underline{\underline{\Phi}}$. 
Finally, by integrating the interfacial power density of internal forces in equation (11) in conjunction with equation (16) and equations (38) and (39) such that,

$$
\Gamma=\int\left[\boldsymbol{\Sigma}^{S}: d \boldsymbol{\epsilon}^{S}+\left(\boldsymbol{\Delta}^{\perp}-\boldsymbol{\Lambda}^{(1)}\right) \cdot d \boldsymbol{\sigma}^{\perp}+\boldsymbol{\Upsilon}^{S}: d \boldsymbol{\epsilon}^{m, S}\right]
$$

we obtain a complementary Shuttleworth equation relating the incoherent surface stress $\Upsilon^{S}$ to the interfacial excess energy $\Gamma$ such that,

$$
\boldsymbol{\Upsilon}^{S}=\left.\frac{\partial \Gamma}{\partial \boldsymbol{\epsilon}^{m, S}}\right|_{\boldsymbol{\epsilon}^{S}, \boldsymbol{\sigma}^{\perp}}-\boldsymbol{\sigma}^{\perp} \cdot \underline{\mathbb{K}}+\underline{\underline{\mathbf{\Phi}}}: \boldsymbol{\epsilon}^{S} .
$$

All together, equations (38), (39) and (41) constitute the generalized Shuttleworth relationship for incoherent interfaces. The coherent surface stress $\boldsymbol{\Sigma}^{S}$ and the transverse excess strain $\boldsymbol{\Delta}^{\perp}$ represent the thermodynamic driving forces deforming the interface while the incoherent surface stress $\Upsilon^{S}$ represents the driving force of stretching one crystal while holding the other fixed and therefore altering the structure of the interface. This generalized formulation not only accounts for the three-dimensional nature of the interface in a Gibbsian sense (2D mathematical object as opposed to an interphase model) but also explicitly considers its interfacial structure. The three thermodynamics quantities described above fully characterize the elastic behavior of coherent and incoherent interfaces under general loading conditions. The extension to curved interfaces will be briefly discussed in section 3.6.

Several differences between the present formulation and that of Cahn and Larché (1982) are observed. Equation (41) is consistent with the incoherent stress introduced in Cahn and Larché when $\boldsymbol{\sigma}^{\perp}=0$ and $\boldsymbol{\epsilon}^{S}=0$. The absence of the in-plane strain in their formulation is essentially due to the fact that they expanded the excess free energy $\Gamma$ in terms of $\boldsymbol{\epsilon}^{S}$ and $\boldsymbol{\epsilon}^{m, S}$ only to the first order (no surface elasticity).

From a surface stress point of view, three major differences need to be noted. First, the coherent and incoherent surface stresses derived in equations (29), (38) 
and (41) reduce in the case of Cahn and Larché (1982) to the residual surface stresses such that,

$$
\Sigma_{C L}^{S}=\boldsymbol{\Gamma}^{(1)} \text { and } \boldsymbol{\Upsilon}_{C L}^{S}=\Upsilon^{(1)}
$$

where the subscript "CL" denotes the Cahn and Larché formulation. Second, the expression for the two-dimensional tensor $\boldsymbol{\Gamma}^{(1)}$ in Cahn and Larché does not account for the contribution attributed to the coupling between the state of coherency, the change in molar volume between the two media and the elastic stiffness of the interface (see second integral in equation (18)). Finally neither the intrinsic coupling between the surface stress and the structural interfacial mismatch (tensor $\underline{\underline{\Phi}}$ ) nor the transverse interfacial behavior (tensors $\mathbb{D}^{\perp}, \underline{\mathbb{H}}$ and $\underline{\mathbb{K}}$ ) are accounted for.

From an interfacial energy point of view, the interfacial power density derived in equation (11) simplifies in the case of Cahn and Larché to,

$$
p_{C L}^{S}=\boldsymbol{\Gamma}^{(1)}: \dot{\boldsymbol{\epsilon}}^{S}+\boldsymbol{\Upsilon}^{(1)}: \dot{\boldsymbol{\epsilon}}^{m, S},
$$

in which neither the surface elasticity $\left(\underline{\underline{\boldsymbol{\Gamma}}}^{(2)}\right)$ nor the couplings both in terms of structural mismatch and transverse behavior are accounted for (see equation (29)). This simplification makes the interfacial excess energy $\Gamma_{C L}$ simply quadratic in terms of the in-plane surface strain $\boldsymbol{\epsilon}^{S}$ and mismatch strain $\boldsymbol{\epsilon}^{m, S}$, while it is shown in equation (16) that the evolution of the interfacial excess energy as a function of the loading path $\left(\boldsymbol{\epsilon}^{S}, \boldsymbol{\sigma}^{\perp}\right)$ and the structural mismatch strain $\boldsymbol{\epsilon}^{m, S}$ is not that straightforward.

\subsection{Discussion on the equilibrium condition of incoherent curved interfaces}

We have intentionally limited ourselves to planar interfaces between two semiinfinite media when dealing with surface excess stresses and surface excess strain in the formulation described above. However, in quite a few practical engineering applications (e.g. composites reinforced with nanoparticles), interfaces can be curved 
surfaces, not planar. In that case, and in the limit where the interface characteristic length scale is much smaller than the interface radius of curvature, the constitutive relationships describing the interface behavior not only need to account for the inplane and transverse behavior (i.e. interfacial Poisson's effect) as well as the interface structural mismatch (as it is described in this manuscript), but also the intrinsic flexural resistance. As mentioned in section 1, few models have been presented in the literature to account for the flexural stiffness (Nozières and Wolf, 1988; Steigmann and Ogden, 1997, 1999; Gao et al., 2014). In this context, the interfacial excess energy is a function of the local in-plane deformation and also comprises a dependence on the curvature introducing an intrinsic flexural resistance of the interface. As described very recently in Gao et al. (2014), the interfacial power density of internal forces for curved interfaces takes the form,

$$
p^{S}=\boldsymbol{\Sigma}^{S}: \dot{\boldsymbol{\epsilon}}^{S}+\boldsymbol{M}^{S}: \dot{\boldsymbol{\kappa}},
$$

where $\boldsymbol{M}^{S}$ is the interface bending moment conjugate of the relative curvature $\boldsymbol{\kappa}$. While accounting for the curvature dependence, these interfacial flexural formulations do not account for the interfacial mismatch or the interfacial transverse behavior (see equation (11)). For curved interfaces, the present formulation would need to include the last term of equation (44) and the interfacial kinematics presented in section 2 would need to be revisited in order to account for the curvature dependence (see for example section 2.2 in Gao et al. (2014)). Additionally, as discussed elsewhere (see equation (5.76) in Dingreville (2007)), due to the "T-decomposition" used in the present formulation, supplemental conditions for the displacement jump across the curved mismatched interface would need to be considered. In other words, an additional incoherent thermodynamic driving force (which could be called the incoherent interfacial bending moment) corresponding to flexural deformation of the 
interface would need to be introduced for curved interfaces. Such an extension of the formulation is beyond the scope of the present manuscript but will be presented in a subsequent study by the authors.

Besides the constitutive relationships describing the interface behavior, the equilibrium condition of curved coherent interfaces is expressed by the generalized YoungLaplace equation and describes the equilibrium relationship between the interface (coherent) stress and the stress in the bulk. For incoherent mismatched curved interfaces, the principle of minimum potential energy can be used to derive the generalized Young-Laplace equation by accounting for (i) the interfacial excess energy, which would depend not only on the interface strains (coherent and incoherent) but also on the interface curvature; (ii) the elastic strain energy of the bulk, which would include the residual elastic field induced by the interfacial excess energy and eigenstrains due to the mismatch across the interface; and (iii) the potential of the external loads. The generalized Young-Laplace equation will also be complemented by the displacement jump condition described in the above paragraph. Again, such an extension of the formulation is beyond the scope of the present manuscript but will be presented in the aforementioned study by the authors. Such a paradigm could be used to fully describe heterogeneous solids containing incoherent curved interfaces and inhomogeneities.

\section{Interfacial isotropic elasticity formulation}

As an illustration of the general formulation presented above, we consider in this section a special case in which both semi-infinite crystals are isotropic elastic materials with the following bulk elastic moduli,

$$
\mathbb{C}_{i j k l, \pm}=\lambda_{ \pm} \delta_{i j} \delta_{k l}+\mu_{ \pm}\left(\delta_{i k} \delta_{j l}+\delta_{i l} \delta_{j k}\right) .
$$




$$
\begin{aligned}
\Gamma_{\alpha \beta}^{(1)} & =\int_{-\infty}^{\infty}\left[\tau_{\alpha \beta}^{S}(\mathbf{x})-\frac{\tau_{3}^{\perp}(\mathbf{x}) \lambda(\mathbf{x})}{\lambda(\mathbf{x})+2 \mu(\mathbf{x})} \delta_{\alpha \beta}\right] d \mathbf{x} \\
& -\int_{-\infty}^{0}\left[\left(\frac{2 \lambda(\mathbf{x}) \mu(\mathbf{x})}{\lambda(\mathbf{x})+2 \mu(\mathbf{x})}-\frac{2 \lambda_{-} \mu_{-}}{\lambda_{-}+2 \mu_{-}}\right) \delta_{\alpha \beta} \epsilon_{\kappa \kappa}^{0, S}+2\left(\mu(\mathbf{x})-\mu_{-}\right) \epsilon_{\alpha \beta}^{0, S}\right] d(5,2) \\
\Gamma_{\alpha \beta \kappa \lambda}^{(2)} & =\lambda^{S} \delta_{\alpha \beta} \delta_{\kappa \lambda}+\mu^{S}\left(\delta_{\alpha \kappa} \delta_{\beta \lambda}+\delta_{\alpha \lambda} \delta_{\beta \kappa}\right) \\
\mathbb{H}_{i \alpha \beta} & =\frac{2 K^{S} \nu^{S}}{E^{\perp}} \delta_{3 i} \delta_{\alpha \beta} \\
\Phi_{\alpha \beta \kappa \lambda} & =\int_{-\infty}^{0}\left[\frac{2 \lambda(\mathbf{x}) \mu(\mathbf{x})}{\lambda(\mathbf{x})+2 \mu(\mathbf{x})} \delta_{\alpha \beta} \delta_{\kappa \lambda}+\mu(\mathbf{x})\left(\delta_{\alpha \kappa} \delta_{\beta \lambda}+\delta_{\alpha \lambda} \delta_{\beta \kappa}\right)\right] g(\mathbf{x}) d \mathbf{x}
\end{aligned}
$$


Taking equations (52)-(55) together with equation (51) gives the in-plane interfacial excess stress as follows

$$
\Sigma_{\alpha \beta}^{S}=\Gamma_{\alpha \beta}^{(1)}-\lambda^{*, S} \delta_{\alpha \beta} \epsilon_{\kappa \kappa}^{m, S}-2 \mu^{*, S} \epsilon_{\alpha \beta}^{m, S}+\lambda^{S} \delta_{\alpha \beta} \epsilon_{\kappa \kappa}^{S}+2 \mu^{S} \epsilon_{\alpha \beta}^{S}+\frac{2 K^{S} \nu^{S}}{E^{\perp}} \sigma_{3}^{\perp} \delta_{\alpha \beta}
$$

where the two new terms compared to Dingreville and Qu (2008) are the ones containing the in-plane structural mismatch embodied by $\epsilon_{\alpha \beta}^{m, S}$. In the case of coherent interfaces, i.e. $\epsilon_{\alpha \beta}^{m, S}=0$, following Cahn and Larché (1982), the formulation developed by Dingreville and Qu (2008) would be sufficient only if both materials have same stress-free molar volumes for which $\epsilon_{\alpha \beta}^{0, S}$ vanishes. In equation (54), $K^{S}=\lambda^{S}+\mu^{S}$ and the surface elastic constants are defined by,

$$
\begin{aligned}
\mu^{S} & =\int_{0}^{\infty}\left(\mu(\mathbf{x})-\mu_{+}\right) d \mathbf{x}+\int_{-\infty}^{0}\left(\mu(\mathbf{x})-\mu_{-}\right) d \mathbf{x} \\
\lambda^{S} & =\int_{0}^{\infty}\left(\frac{2 \lambda(\mathbf{x}) \mu(\mathbf{x})}{\lambda(\mathbf{x})+2 \mu(\mathbf{x})}-\frac{2 \lambda_{+} \mu_{+}}{\lambda_{+}+2 \mu_{+}}\right) d \mathbf{x} \\
& +\int_{-\infty}^{0}\left(\frac{2 \lambda(\mathbf{x}) \mu(\mathbf{x})}{\lambda(\mathbf{x})+2 \mu(\mathbf{x})}-\frac{2 \lambda_{-} \mu_{-}}{\lambda_{-}+2 \mu_{-}}\right) d \mathbf{x} \\
\mu^{*, S} & =\int_{-\infty}^{0} \mu(\mathbf{x}) g(\mathbf{x}) d \mathbf{x}, \\
\lambda^{*, S} & =\int_{-\infty}^{0} \frac{2 \lambda(\mathbf{x}) \mu(\mathbf{x})}{\lambda(\mathbf{x})+2 \mu(\mathbf{x})} g(\mathbf{x}) d \mathbf{x}, \\
\frac{2 K^{S} \nu^{S}}{E^{\perp}} & =\int_{0}^{\infty}\left(\frac{\lambda(\mathbf{x})}{\lambda(\mathbf{x})+2 \mu(\mathbf{x})}-\frac{\lambda_{+}}{\lambda_{+}+2 \mu_{+}}\right) d \mathbf{x} \\
& +\int_{-\infty}^{0}\left(\frac{\lambda(\mathbf{x})}{\lambda(\mathbf{x})+2 \mu(\mathbf{x})}-\frac{\lambda_{-}}{\lambda_{-}+2 \mu_{-}}\right) d \mathbf{x} .
\end{aligned}
$$

\footnotetext{
Through this exercise several points are noteworthy:
}

- In equation (56), $\lambda^{S}$ and $\mu^{S}$, which could be defined as the surface Lamé constants, are consistent (in terms of their definition and analytical expressions) with other classical formulations derived by many authors for surface stress in 
solids (Gurtin and Murdoch, 1975; Gurtin et al., 1998; Sharma et al., 2003; Duan et al., 2005; Chen et al., 2007; Duan et al., 2007).

- The effective transverse surface "Poisson's ratio" $2 K^{S} \nu^{S} / E^{\perp}$ in equation (61) is consistent with the formulation for an interface containing no mismatch and stress-free strains (Dingreville and Qu, 2008).

- The present formulation introduces two new surface constants $\mu^{*, S}$ and $\lambda^{*, S}$ defined in equations (59)-(60). These constants are solely related to the interfacial incoherency and the in-plane structural mismatch strain $\epsilon_{\alpha \beta}^{m, S}$ and correspond to a correction to the interfacial residual stress $\Gamma_{\alpha \beta}^{(1)}$. To our knowledge, their expressions and physical origins are presented for the first time in the literature.

With this in mind, the in-plane interfacial excess stress components yield

$$
\begin{aligned}
& \Sigma_{11}^{S}=\Gamma_{11}^{(1)}-\lambda^{*, S} \epsilon_{\kappa \kappa}^{m, S}-2 \mu^{*, S} \epsilon_{11}^{m, S}+\lambda^{S} \epsilon_{\kappa \kappa}^{S}+2 \mu^{S} \epsilon_{11}^{S}+\frac{2 K^{S} \nu^{S}}{E^{\perp}} \sigma_{3}^{\perp}, \\
& \Sigma_{22}^{S}=\Gamma_{22}^{(1)}-\lambda^{*, S} \epsilon_{\kappa \kappa}^{m, S}-2 \mu^{*, S} \epsilon_{22}^{m, S}+\lambda^{S} \epsilon_{\kappa \kappa}^{S}+2 \mu_{S} \epsilon_{22}^{S}+\frac{2 K^{S} \nu^{S}}{E^{\perp}} \sigma_{3}^{\perp}, \\
& \Sigma_{12}^{S}=\Gamma_{12}^{(1)}-2 \mu^{*, S} \epsilon_{12}^{m, S}+2 \mu^{S} \epsilon_{12}^{S} .
\end{aligned}
$$

Thus, from equations (62)-(64), $\mu^{S}$ and $\mu^{*, S}$ are in-plane interfacial shear moduli associated with in-plane interfacial shear strain $\epsilon_{12}^{S}$ and in-plane incoherent mismatched shear strain $\epsilon_{12}^{m, S}$ respectively. The surface elastic moduli $\lambda^{S}$ and $\lambda^{*, S}$ are in-plane interfacial Lamé constants. Furthermore, the in-plane biaxial hydrostatic stress is given by $\Sigma_{\alpha \alpha}^{S} / 2$ where

$$
\Sigma_{\alpha \alpha}^{S}=\Gamma_{\alpha \alpha}^{(1)}-2 K^{*, S} \epsilon_{\kappa \kappa}^{m, S}+2 K^{S} \epsilon_{\kappa \kappa}^{S}+\frac{4 K^{S} \nu^{S}}{E^{\perp}} \sigma_{3}^{\perp}
$$

${ }_{448}$ where $K^{S *}=\mu^{*, S}+\lambda^{*, S}$ can be interpreted as the in-plane bi-axial surface modulus of 
the incoherent interface associated with the structural mismatch (or deviation from coherency) $\epsilon_{\kappa \kappa}^{m, S}$.

\subsection{Transverse interfacial excess strain}

Regarding the transverse interfacial excess strain, using equations (35) along with (46)-(50), we find the interfacial excess strain,

$$
\Delta_{i}^{\perp}=\Lambda_{i}^{(1)}+\mathbb{K}_{i \alpha \beta} \epsilon_{\alpha \beta}^{m, S}+\Lambda_{i j}^{(2)} \sigma_{j}^{\perp}-\mathbb{H}_{i \alpha \beta} \epsilon_{\alpha \beta}^{S}
$$

with the transverse surface tensors defined by,

$$
\begin{aligned}
\Lambda_{i}^{(1)} & =\int_{-\infty}^{\infty}\left[\frac{\lambda(\mathbf{x})+\mu(\mathbf{x})}{(\lambda(\mathbf{x})+2 \mu(\mathbf{x})) \mu(\mathbf{x})} \delta_{3 i} \tau_{3}^{\perp}(\mathbf{x})-\frac{1}{\mu(\mathbf{x})} \tau_{i}^{\perp}(\mathbf{x})\right] d \mathbf{x} \\
& +\int_{-\infty}^{0}\left[\left(\frac{\lambda(\mathbf{x})}{\lambda(\mathbf{x})+2 \mu(\mathbf{x})}-\frac{\lambda_{-}}{\lambda_{-}+2 \mu_{-}}\right) \delta_{3 i} \epsilon_{\kappa \kappa}^{0, S}\right] d \mathbf{x} \\
& +\int_{-\infty}^{0}\left[\epsilon_{i}^{\perp \star}(\mathbf{x})-\epsilon_{i}^{\perp 0}\right] d \mathbf{x}, \\
\Lambda_{i j}^{(2)} & =\left(\frac{1}{E^{\perp}}-\frac{1}{\mu^{\perp}}-\frac{4 K^{S} \nu^{S^{2}}}{E^{\perp}}\right) \delta_{3 i} \delta_{3 j}+\frac{1}{\mu^{\perp}} \delta_{i j}, \\
\mathbb{K}_{i \alpha \beta} & =\int_{-\infty}^{0} \frac{\lambda(\mathbf{x})}{\lambda(\mathbf{x})+2 \mu(\mathbf{x})} g(\mathbf{x}) \delta_{3 i} \delta_{\alpha \beta} d \mathbf{x} .
\end{aligned}
$$

Similarly to the formulation for the surface stress, taking equation (66) together with the expressions for the transverse surface tensors yields

$$
\Delta_{i}^{\perp}=\Lambda_{i}^{(1)}+\frac{2 K^{*, S} \nu^{*, S}}{E^{*, \perp}} \delta_{3 i} \epsilon_{\kappa \kappa}^{m, S}+\left(\frac{1}{E^{\perp}}-\frac{1}{\mu^{\perp}}-\frac{4 K^{S} \nu^{S^{2}}}{E^{\perp^{2}}}\right) \delta_{3 i} \sigma_{3}^{\perp}+\frac{1}{\mu^{\perp}} \sigma_{i}^{\perp}-\frac{2 K^{S} \nu^{S}}{E^{\perp}} \delta_{3 i} \epsilon_{\kappa \kappa}^{S},
$$

where the transverse elastic constant associated with the structural mismatch can be defined as,

$$
\frac{2 K^{*, S} \nu^{*, S}}{E^{*, \perp}}=\int_{-\infty}^{0} \frac{\lambda(\mathbf{x})}{\lambda(\mathbf{x})+2 \mu(\mathbf{x})} g(\mathbf{x}) d \mathbf{x}
$$


and,

$$
\begin{aligned}
\frac{1}{\mu^{\perp}} & =\int_{0}^{\infty}\left[\frac{1}{\mu(\mathbf{x})}-\frac{1}{\mu_{+}}\right] d \mathbf{x}+\int_{-\infty}^{0}\left[\frac{1}{\mu(\mathbf{x})}-\frac{1}{\mu_{-}}\right] d \mathbf{x} \\
\frac{1}{E^{\perp}}-\frac{4 K^{S} \nu^{S^{2}}}{E^{\perp^{2}}} & =\int_{0}^{\infty}\left[\frac{1}{\lambda(\mathbf{x})+2 \mu(\mathbf{x})}-\frac{1}{\lambda_{+}+2 \mu_{+}}\right] d \mathbf{x} \\
& +\int_{-\infty}^{0}\left[\frac{1}{\lambda(\mathbf{x})+2 \mu(\mathbf{x})}-\frac{1}{\lambda_{-}+2 \mu_{-}}\right] d \mathbf{x}
\end{aligned}
$$

460 461

462

It follows that the components of the interfacial transverse excess strain can be expressed as,

$$
\begin{aligned}
& \Delta_{1}^{\perp}=\Lambda_{1}^{(1)}+\frac{1}{\mu^{\perp}} \sigma_{1}^{\perp} \\
& \Delta_{2}^{\perp}=\Lambda_{2}^{(1)}+\frac{1}{\mu^{\perp}} \sigma_{2}^{\perp} \\
& \Delta_{3}^{\perp}=\Lambda_{3}^{(1)}+2 K^{*, S}\left(\frac{\nu^{*, S}}{E^{*, \perp}}-\frac{\nu^{S}}{E^{\perp}}\right) \epsilon_{\kappa \kappa}^{m, S}+\frac{1}{E^{\perp}}\left(\sigma_{3}^{\perp}-\nu^{S}\left(\Sigma_{\alpha \alpha}^{S}-\Gamma_{\alpha \alpha}^{(1)}\right)\right) .
\end{aligned}
$$

It is again noteworthy to point out that $\Lambda_{i}^{(1)}$ and the second term in $\Delta_{3}^{\perp}$ are new when compared to Dingreville and $\mathrm{Qu}$ (2008). Their origin stems from the (stress-free) eigenstrains and structural mismatch for incoherent interfaces, i.e. for $\epsilon_{\kappa \kappa}^{m, S} \neq 0$.

\subsection{Interfacial thermo-elastic properties}

Of particular interest (see for example the Levin's formula and Hill's connections problems in Duan and Karihaloo (2007)) is the case for which we assume that the interfacial incoherency is due to thermal strains associated with an isotropic in-plane interfacial coefficient of thermal expansion (CTE) denoted $\alpha^{S}$, i.e.

$$
\epsilon_{\alpha \beta}^{m, S}=\alpha^{S} \delta_{\alpha \beta} \Delta T
$$


where $\Delta T$ is the temperature difference. In the bulk of both media, assuming an isotropic CTE, the eigenstrain $\epsilon^{0, S}$ can simply be expressed by,

$$
\epsilon_{\alpha \beta}^{0, S}=\left(\alpha_{-}-\alpha_{+}\right) \delta_{\alpha \beta} \Delta T,
$$

where $\alpha_{-}$and $\alpha_{+}$are the bulk CTEs for media "-" and "+" respectively while the reference medium is assumed to be the medium "+".

Thus, the expression for the isotropic surface stress in equation (56) reduces to

$$
\Sigma_{\alpha \beta}^{S}=\Gamma_{\alpha \beta}^{(1)}-d_{0}^{*} \delta_{\alpha \beta} \Delta T+\lambda^{S} \delta_{\alpha \beta} \epsilon_{\kappa \kappa}^{S}+2 \mu^{S} \epsilon_{\alpha \beta}^{S}+\frac{2 K^{S} \nu^{S}}{E^{\perp}} \sigma_{3}^{\perp} \delta_{\alpha \beta},
$$

and,

$$
d_{0}^{*}=2\left(\lambda^{*, S}+\mu^{*, S}\right) \alpha^{S}=2 K^{*, S} \alpha^{S} .
$$

The present formulation sheds light on the in-plane thermal-mechanical connection of the interface and its elastic origin assuming a resulting incoherent interface due to temperature difference. This thermal-mechanical relationship is however different from that of Murdoch $(1976,2005)$ and Duan and Karihaloo (2007). For instance, Duan and Karihaloo (2007) reported the following equation (see equation (3) in their paper):

$$
\Sigma_{\alpha \beta}^{S}=\lambda^{S} \delta_{\alpha \beta} \epsilon_{\kappa \kappa}^{S}+2 \mu^{S} \epsilon_{\alpha \beta}^{S}-d_{0} \delta_{\alpha \beta} \Delta T,
$$

where $d_{0}$ is assumed by the authors to be $d_{0}=2\left(\lambda^{S}+\mu^{S}\right) \alpha^{S}$. Several differences are observed. First, the contribution of the residual surface stress $\Gamma_{\alpha \beta}^{(1)}$ and the contribution of the transverse stress are not taken into account in Duan and Karihaloo. This is due to the choice of the interfacial constitutive behavior used in their paper. Second, the contribution due to the interfacial mismatch through the term $\left(\lambda^{*, S}+\mu^{*, S}\right)$ (as defined by our equations (59) and (60)) is arbitrarily taken as $\left(\lambda^{S}+\mu^{S}\right)$ in Duan and Karihaloo. In the present formulation, the interpretation 
of the thermal-mechanical connection $d_{0}^{*}$ is unambiguously and explicitly associated with the structural mismatch at the interface coming from temperature change. In turn, this can ultimately be related to interfacial defects and associated deformation fields. This is not the case for $d_{0}$ in the formulation of Duan and Karihaloo which appears to be empirically introduced. Note here that the thermo-elastic coupling is spatially defined at every vector position starting from $\mathbf{x}=0$ to a few atomic distance close to the interface in medium "-".

Additionally, to complete the description of the thermo-elasticity of the interface, the interfacial constitutive behavior is also accompanied by equation (70) which reduces to:

$\Delta_{i}^{\perp}=\Lambda_{i}^{(1)}+\frac{2 d_{0}^{*} \nu^{*, S}}{E^{*, \perp}} \delta_{3 i} \Delta T+\left(\frac{1}{E^{\perp}}-\frac{1}{\mu^{\perp}}-\frac{4 K^{S} \nu^{S^{2}}}{E^{\perp^{2}}}\right) \delta_{3 i} \sigma_{3}^{\perp}+\frac{1}{\mu^{\perp}} \sigma_{i}^{\perp}-\frac{2 K^{S} \nu^{S}}{E^{\perp}} \delta_{3 i} \epsilon_{\kappa \kappa}^{S}$.

In particular, the transverse excess strain along the normal to the interface is given by,

$$
\Delta_{3}^{\perp}=\Lambda_{3}^{(1)}+\frac{2 d_{0}^{*} \nu^{*, S}}{E^{*, \perp}} \Delta T+\frac{1}{E^{\perp}}\left(1-\frac{4 K^{S} \nu^{S^{2}}}{E^{\perp^{2}}}\right) \sigma_{3}^{\perp}-\frac{2 K^{S} \nu^{S}}{E^{\perp}} \epsilon_{\kappa \kappa}^{S} .
$$

Equations (82) and (83) are extensions of Dingreville and Qu (2008) to thermoelastic interfaces and were not considered in the works of Murdoch (1976, 2005) and Duan and Karihaloo (2007).

\subsection{Insight on interfacial elasticity}

An important point is raised in the sections above: deforming a coherent or incoherent interface does not always increase its interfacial excess energy. Indeed, the interfacial excess energy decreases if a deformation path reduces the interface coherent surface, the interfacial excess strain, or the interface incoherent stress. This assertion can have important consequences for problems in which the incorporation 
of interfacial properties play an important role (Cammarata, 1994; Chen et al., 2007; Duan and Karihaloo, 2007). Indeed, the in-plane strain, transverse stress and mismatch strain can be considered as three process control variables (with the first two being associated with the deformation path and the latter one being associated with the interface structure) that can accentuate or minimize the role of interfacial elasticity.

For instance, in the case of thermo-elasticity provided in section 4.3, the loading space for which the coherent surface stress $\boldsymbol{\Sigma}^{S}$ and the interfacial excess strain $\boldsymbol{\Delta}^{\perp}$ vanish is of particular interest. Using equation (79) for the biaxial surface stress $\left(\epsilon_{11}^{S}=\epsilon_{22}^{S}=\epsilon^{S}\right)$ and equation (83) for the transverse interfacial excess strain, this configuration would correspond to the loading path $\left(\epsilon^{S}, \sigma^{\perp}\right)$ satisfying the following equation:

$$
\left[\begin{array}{c}
\frac{\Gamma_{11}^{(1)}}{2} \\
\Lambda_{3}^{(1)}
\end{array}\right]+\left[\begin{array}{ccc}
2 K^{S} & \frac{2 K^{S} \nu^{S}}{E^{\perp}} & -d_{0}^{*} \\
-\frac{4 K^{S} \nu^{S}}{E^{\perp}} & \frac{1}{E^{\perp}}\left(1-\frac{4 K^{S} \nu^{S^{2}}}{E^{\perp^{2}}}\right) & \frac{2 d_{0}^{*} \nu^{*, S}}{E^{*, \perp}}
\end{array}\right] \cdot\left[\begin{array}{c}
\epsilon^{S} \\
\sigma^{\perp} \\
\Delta T
\end{array}\right]=\left[\begin{array}{l}
0 \\
0
\end{array}\right] .
$$

Equation (84) can be parametrized to obtain the loading path line in the $\left(\epsilon^{S}, \sigma^{\perp}\right)$ space as a function of the interfacial elastic constants $\left(K^{S}, \nu^{S}, E^{\perp}, \nu^{*, S}, E^{*, \perp}, d_{0}^{*}\right)$ and the temperature difference $\Delta T$. Clearly, this illustrates the concept that one can construct a loading path that would minimize the impact of the interface on the behavior of a material system separated by it or construct a loading path for which the surface to volume ratio is significant.

\section{Illustration from atomistic simulations of $\mathrm{Cu} / \mathrm{Cu}_{2} \mathrm{O}$ interfaces}

As discussed in the previous sections, the interfacial excess energy is a function of the in-plane surface strain, the transverse stress and the interface mismatch strain. 
In this section we illustrate the formulation developed above with examples based on atomistic simulations for incoherent interfaces between $\mathrm{Cu}$ and its oxide $\mathrm{Cu}_{2} \mathrm{O}$ under various loading configurations.

\subsection{Atomistic model for $\mathrm{Cu}(111) / C u_{2} \mathrm{O}(111)$ interface and simulation methodology}

To demonstrate the generalized formulation of a mismatched interface from a discrete medium point of view, we consider as an example a flat incoherent interface between $\mathrm{Cu}$ and its oxide $\mathrm{Cu}_{2} \mathrm{O}$. As shown in Fig. 3, the crystallographic orientation used for the present atomistic simulations is chosen as the most probable observed interface between $\mathrm{Cu}$ and its oxide $\mathrm{Cu}_{2} \mathrm{O}$ (Dubois, 1982; Jensen et al., 1991; Werner et al., 1997; Schedel-Niedrig et al., 2000; Pierson et al., 2005; Soon et al., 2006). As such, we consider $\mathrm{Cu}$ and $\mathrm{Cu}_{2} \mathrm{O}$ blocks oriented in the (111) direction when the interface is created. Note that these surfaces are isotropic in the plane of the interface.

The interatomic potential based on pairwise interactions proposed by Hallil et al. (2014) is used to describe the $\mathrm{Cu}_{2} \mathrm{O}$ properties while the embedded-atom method (EAM) potential developed by Cleri and Rosato (1993) is employed for the Cu metallic phase. The cross atomic interactions between $\mathrm{Cu}$ and $\mathrm{Cu}_{2} \mathrm{O}$ is modeled through (i) a 9-6 Lennard-Jones (LJ) potential for the interaction between copper metallic atoms and copper atoms from the oxide, and (ii) a Morse potential linking copper metallic atoms with the oxygen atoms. Note that there are two types of metallic bonds in this model: the $\mathrm{Cu}-\mathrm{Cu}$ non-bond interaction in the copper oxide which is modeled by the 9-6 LJ potential and the $\mathrm{Cu}-\mathrm{Cu}$ metallic bond modeled by EAM potential. The bond interaction between the oxygen and copper species defined by the Morse potential remains the same whether the $\mathrm{Cu}$ atoms belong either to the pure copper phase or the copper oxide compounds (Hallil et al., 2014). 
Various interfacial structural mismatches are considered by varying the overlap between the surface lattice vectors of each material during the matching process between both phases. Matching $\mathrm{Cu}$ and $\mathrm{Cu}_{2} \mathrm{O}$ blocks presents some issues in terms of coherency since the $\mathrm{Cu}_{2} \mathrm{O}(111)$ unit cell $\left(\ell_{\mathrm{Cu}_{2} \mathrm{O}}=6.04825 \AA\right)$ is larger than the $\mathrm{Cu}$ (111) unit cell $\left(\ell_{\mathrm{Cu}}=2.5542 \AA\right)$. Following equation (6), in this atomistic context, the structural mismatch is simply defined as,

$$
\epsilon_{\alpha \beta}^{m, S}=2 \frac{n \ell_{\mathrm{Cu}}-m \ell_{\mathrm{Cu}_{2} \mathrm{O}}}{n \ell_{\mathrm{Cu}}+m \ell_{\mathrm{Cu}_{2} \mathrm{O}}} \delta_{\alpha \beta}
$$

where $\ell_{\mathrm{Cu}}$ and $\ell_{\mathrm{Cu}_{2} \mathrm{O}}$ are the lattice constants of $\mathrm{Cu}$ and $\mathrm{Cu}_{2} \mathrm{O}$ respectively and $(n, m)$ are scaling factors (taken as integers) used to obtain various mismatch strains. To minimize the structural mismatch, several sets of couple $(m, n)$ have been chosen such that the overall strain is small when the interface is formed.

The interface model dimensions and mismatch strains simulated are summarized in Table 1. A typical computation cell contains on average 20,000 atoms composed of $N_{\mathrm{Cu}}$ atoms in the $\mathrm{Cu}$ phase and $N_{\mathrm{Cu}_{2} \mathrm{O}}$ atoms in the $\mathrm{Cu}_{2} \mathrm{O}$ phase. Periodic boundary conditions are used in all directions. Note that the use of periodic boundary conditions in the transverse direction implicitly introduces a second interface at the edge of the calculation box with an identical structure as the interface located at the center of atomic assembly. Dimensions of the simulation box $(W, D, L)$ have been chosen such that atoms far away from the interface have energy levels corresponding to the perfect bulk configuration. In other words, the two interfaces present in the atomistic system do not interact, therefore avoiding the size effect mentioned in section 2 .

Once the initial interfacial assembly is created, a molecular statics calculation, which employs a nonlinear conjugate gradient method, is used to determine the minimum energy interface configuration (i.e., relaxed positions of the atoms) and 
to find the self equilibrium state of the interface. Due to the in-plane periodicity, relaxation of the structure occurs only in the direction perpendicular to the boundary plane. The transverse stress in the relaxed configuration is therefore null. A detailed discussion of interface atomistic structural rearrangement throughout the entire simulation process and resulting interfacial point defects is beyond the scope of the present manuscript.

After the minimum interfacial energy configuration is obtained, the interfacial assembly is elastically deformed either by applying a transverse tensile stress $\sigma_{3}^{\perp}$ or by applying biaxial strains $\epsilon_{11}^{S}=\epsilon_{22}^{S}=\epsilon^{S}$ of a couple percents in the interfacial plane. Each of these stresses and biaxial strains is less than the critical load required for defect nucleation (dislocation notably) for the interfaces considered in this work, so that we remain in the elastic domain. In the configuration when the tensile stress is applied, the $x$ - and $y$-direction boundaries are specified as stress-free throughout deformation.

For each deformation process described in the above, MS simulations are performed to compute the interfacial excess energy at the equilibrium state of the system. In the case of the discrete atomistic systems used in this work, the integral of the energy profile defined in equation (12) is discretized into a summation over a set of atoms within each region, rigorously separating the energy profile normal to the interface into two regions (Dingreville and Qu, 2009) such that,

$$
\Gamma=\frac{1}{2 A_{0}}\left[\sum_{i=1}^{N_{\mathrm{Cu}}}\left(e^{(i)}(z)-e_{\mathrm{Cu}}^{(0)}\right)+\sum_{i=1}^{N_{\mathrm{Cu}_{2} \mathrm{O}}}\left(e^{(i)}(z)-e_{\mathrm{Cu}_{2} \mathrm{O}}^{(0)}\right)\right],
$$

where $e^{(i)}$ is the total energy of atom $i$ enclosed by the area $A_{0}$ on the interface, $N_{\mathrm{Cu}}$ and $N_{\mathrm{Cu}_{2} \mathrm{O}}$ are the number of atoms in the copper region and its oxide respectively, and $e_{\mathrm{Cu}}^{(0)}$ and $e_{\mathrm{Cu}_{2} \mathrm{O}}^{(0)}$ are the total energies of an atom in a perfect lattice (bulk config- 
uration) far away from the surface in medium $\mathrm{Cu}$ and medium $\mathrm{Cu}_{2} \mathrm{O}$, respectively. The bulk energies, $e_{\mathrm{Cu}}^{(0)}$ and $e_{\mathrm{Cu}_{2} \mathrm{O}}^{(0)}$, are determined by averaging the total energy $e^{(i)}$ of a group of atoms positioned sufficiently far away from the interface such that the presence of the boundary can be ignored. Using $e_{\mathrm{Cu}}^{(0)}$ and $e_{\mathrm{Cu}_{2} \mathrm{O}}^{(0)}$, an excess energy is computed for every atom within the interface model; the sum of these excess energies is defined as the total interface energy. Note that the factor 2 in the denominator of equation (86) accounts for the presence of the two actual interfaces within the atomic assembly due to the periodic boundary conditions.

\subsection{Comparisons between the discrete atomistic simulations and the generalized Shuttleworth relationships}

The above procedure has been used to calculate the variation of the interfacial excess energy under biaxial straining or transverse loading. The interfacial elastic properties used for the comparison between the atomistic simulations and the theoretical model presented in the above are listed in Table 2 . These interfacial elastic properties have been obtained numerically using a least-square method accounting for the various loading paths used the atomistic system based on equations (87)(89), thus limiting the number of interfacial elastic constants that can be calculated through this method. Note that the semi-analytical methodology developed by Dingreville and $\mathrm{Qu}$ (2009) in the case of the EAM potential could be used as an alternative to derive the interfacial properties. However this formulation would need to be modified to account for the complexity of the two interatomic potentials and cross atomic interactions used in this manuscript for the $\mathrm{Cu} / \mathrm{Cu}_{2} \mathrm{O}$ interfacial system. Such modifications of the methodology are beyond the scope of the present manuscript.

Looking first at the interfacial excess energy as a function of the mismatch strain 
$\boldsymbol{\epsilon}^{m, S}$ when no load is applied $\left(\boldsymbol{\epsilon}^{S}=0\right.$ and $\left.\boldsymbol{\sigma}^{\perp}=0 \mathrm{MPa}\right)$, equation (16) reduces to

$$
\left.\Gamma\right|_{\boldsymbol{\epsilon}^{S}=0, \boldsymbol{\sigma}^{\perp}=0}=\Gamma^{*}=\Gamma_{0}+\Upsilon_{\alpha \alpha}^{(1)} \epsilon_{\alpha \alpha}^{m, S}+\frac{1}{2} \epsilon_{\alpha \alpha}^{m, S} \Upsilon_{\alpha \alpha \beta \beta}^{(2)} \epsilon_{\beta \beta}^{m, S}
$$

The MS simulation results for the variation of the interfacial excess energy as a function of the mismatch strain are shown in Fig. 4 together with the results from the present continuum model. It is seen that these results agree very well with those obtained by the generalized interfacial formulation and show a quadratic behavior.

Considering now the variation of interfacial excess energy as a function of the transverse stress $\boldsymbol{\sigma}^{\perp}$ for various mismatch strains (no in-plane strain applied), the interfacial excess energy $\Gamma$ in equation (16) reduces to

$$
\left.\Gamma\right|_{\boldsymbol{\epsilon}^{S}=0}=\Gamma^{*}+\frac{1}{2} \sigma_{3}^{\perp} \Lambda_{33}^{(2)} \sigma_{3}^{\perp}
$$

We again observe a very good agreement between the atomistic simulation results and the present theoretical formulation as it is plotted in Fig. 5.

Similarly, in the case of the variation of interfacial excess energy as a function of the biaxial strain $\boldsymbol{\epsilon}^{S}$ (no transverse stress applied), equation (16) reduces to

$$
\left.\Gamma\right|_{\sigma^{\perp}=0}=\Gamma^{*}+\Gamma_{\alpha \alpha}^{(1)} \epsilon_{\alpha \alpha}^{S}+\frac{1}{2} \epsilon_{\alpha \alpha}^{S} \Gamma_{\alpha \alpha \beta \beta}^{(2)} \epsilon_{\beta \beta}^{S}-\epsilon_{\alpha \alpha}^{m, S} \Phi_{\alpha \alpha \beta \beta} \epsilon_{\beta \beta}^{S}
$$

Comparison between the atomistic simulations and the theoretical models are presented in Fig. 6. The results of the present model are in good agreement with those obtained by full-blown atomistic simulations.

\section{Conclusion}

The main contribution of this manuscript is the derivation of a generalized continuum framework describing the elastic behavior of coherent and incoherent interfaces 
under general loading conditions based on the Gibbs dividing surface concept combined with the so-called "T-decomposition". This generalized Shuttleworth-Herring formulation considers the equilibrium of mismatched interfaces as a function of the in-plane strain $\boldsymbol{\epsilon}^{S}$, the transverse stress $\boldsymbol{\sigma}^{\perp}$ and the structural mismatch strain $\boldsymbol{\epsilon}^{m, S}$. This formulation not only accounts for the three-dimensional nature of the interface in a Gibbsian sense (2D mathematical object as opposed to an interphase model) but also explicitly considers its interfacial structure. Two surface stresses are thus introduced: a coherent surface stress $\boldsymbol{\Sigma}^{S}$ and an incoherent surface stress $\Upsilon^{S}$, along with a transverse excess strain $\Delta^{\perp}$. The coherent surface stress $\Sigma^{S}$ and the transverse excess strain $\Delta^{\perp}$ represent the thermodynamic driving forces of stretching the interface while the incoherent surface stress $\mathbf{\Upsilon}^{S}$ represents the driving force of altering the structure of the interface by stretching one crystal while holding the other fixed. These three quantities fully characterize the elastic behavior of coherent and incoherent interfaces and read:

$$
\begin{aligned}
\boldsymbol{\Sigma}^{S} & =\left.\frac{\partial \Gamma}{\partial \boldsymbol{\epsilon}^{S}}\right|_{\boldsymbol{\epsilon}^{m, S}, \boldsymbol{\sigma}^{\perp}}+\boldsymbol{\sigma}^{\perp} \cdot \underline{\mathbb{H}}, \\
\boldsymbol{\Delta}^{\perp}-\boldsymbol{\Lambda}^{(1)} & =\left.\frac{\partial \Gamma}{\partial \boldsymbol{\sigma}}\right|_{\boldsymbol{\epsilon}^{S}, \boldsymbol{\epsilon}^{m, S}}-\underline{\mathbb{H}}: \boldsymbol{\epsilon}^{S}+\underline{\mathbb{K}}: \boldsymbol{\epsilon}^{m, S}, \\
\mathbf{\Upsilon}^{S} & =\left.\frac{\partial \Gamma}{\partial \boldsymbol{\epsilon}^{m, S}}\right|_{\boldsymbol{\epsilon}^{S}, \boldsymbol{\sigma}^{\perp}}-\boldsymbol{\sigma}^{\perp} \cdot \underline{\mathbb{K}}+\underline{\underline{\mathbf{\Phi}}}: \boldsymbol{\epsilon}^{S} .
\end{aligned}
$$

This formulation naturally introduces interfacial elastic tensors, as well as an implicit coupling between the interfacial structure and interfacial stresses. Three surface tensors $\left(\underline{\underline{\Gamma}}^{(2)}, \Lambda^{(2)}, \underline{\mathbb{H}}\right)$ describe the pure elastic behavior of the interface (i.e., independent of the structural mismatch) while four surface tensors $\left(\Gamma^{(1)}, \Lambda^{(1)}, \underline{\underline{\Phi}}\right.$, $\underline{\mathbb{K}}$ ) describe and/or account for the coupling between the interface and the state of coherency (i.e. dependent on the structural mismatch).

The isotropic case is developed in detail and particular attention is paid to the 
case of interfacial thermo-elasticity resulting from the mismatch of coefficient of thermal expansions between two dissimilar materials. This exercise provides some insight on the physical significance of the interfacial elastic constants introduced in the formulation and illustrates the obvious coupling between the interface structure and its associated thermodynamics quantities.

Comparisons between discrete atomistic simulations for incoherent interfaces between $\mathrm{Cu}$ and its oxide $\mathrm{Cu}_{2} \mathrm{O}$ and the present continuum formulation demonstrate that this generalized framework for mismatched interfaces successfully characterizes the elastic behavior of such interfaces.

\section{Acknowledgments}

A.H. would like to thank the UMI GTL/CNRS (UMI 2958) and Pr. M. Cherkaoui for their valuable support for part of this work along with the support from the European Commission for partial funding of this work under the "NanoInterface" project (NMP-2008-214371). S.B. would also like to thank the support of the French government through the National Research Agency (ANR) under the program "Investment in the future" (Labex DAMAS referenced as ANR-11-LABX-0008-01).

Sandia is a multiprogram laboratory operated by Sandia Corporation, a Lockheed Martin Company, for the United States Department of Energy, under Contract No. DE-AC04-94AL85000. 


\section{References}

Berbenni, S., Cherkaoui, M., 2010. Homogenization of multicoated inclusionreinforced linear elastic composites with eigenstrains: Application to thermoelastic behavior. Philosophical Magazine 90 (22), 3003-3026.

Cahn, J. W., Larché, F., 1982. Surface stress and the chemical equilibrium of small crystals-II. Solid particles embedded in a solid matrix. Acta Metallurgica 30 (1), $51-56$.

Cammarata, R. C., 1994. Surface and interface stress effects in thin films. Progress in Surface Science 46 (1), 1-38.

Cammarata, R. C., 1997. Surface and interface stress effects on interfacial and nanostructured materials. Materials Science and Engineering: A 237 (2), 180-184.

Cammarata, R. C., Sieradzki, K., 1994. Surface and interface stresses. Annual Review of Materials Science 24 (1), 215-234.

Cammarata, R. C., Sieradzki, K., Spaepen, F., 2000. Simple model for interface stresses with application to misfit dislocation generation in epitaxial thin films. Journal of Applied Physics 87 (3), 1227-1234.

Chen, T., Dvorak, G. J., Yu, C. C., 2007. Solids containing spherical nano-inclusions with interface stresses: effective properties and thermal-mechanical connections. International Journal of Solids and Structures 44 (3-4), 941-955.

Cleri, F., Rosato, V., 1993. Tight-binding potentials for transition metals and alloys. Physical Review B 48 (1), 22-33. 
Dingreville, R., 2007. Modeling and characterization of the elastic behavior of interfaces in nanostructured materials: from an atomistic description to a continuum approach. Ph.D. thesis, Georgia Institute of Technology.

Dingreville, R., Qu, J., 2007. A semi-analytical method to compute surface elastic properties. Acta Materialia 55 (1), 141-147.

Dingreville, R., Qu, J., 2008. Interfacial excess energy, excess stress and excess strain in elastic solids: Planar interfaces. Journal of the Mechanics and Physics of Solids 56 (5), 1944-1954.

Dingreville, R., Qu, J., 2009. A semi-analytical method to estimate interface elastic properties. Computational Materials Science 46 (1), 83-91.

Dingreville, R., Qu, J., Cherkaoui, M., 2005. Surface free energy and its effect on the elastic behavior of nano-sized particles, wires and films. Journal of the Mechanics and Physics of Solids 53 (8), 1827-1854.

Duan, H. L., Karihaloo, B. L., 2007. Thermo-elastic properties of heterogeneous materials with imperfect interfaces: Generalized Levin's formula and Hill's connections. Journal of the Mechanics and Physics of Solids 55 (5), 1036-1052.

Duan, H. L., Wang, J., Huang, Z. P., Karihaloo, B. L., 2005. Size-dependent effective elastic constants of solids containing nano-inhomogeneities with interface stress. Journal of the Mechanics and Physics of Solids 53 (7), 1574-1596.

Duan, H. L., Yi, X., Huang, Z. P., Wang, J., 2007. A unified scheme for prediction of effective moduli of multiphase composites with interface effects. Part I: Theoretical framework. Mechanics of Materials 39 (1), 81-93. 
Dubois, L. H., 1982. Oxygen chemisorption and cuprous oxide formation on $\mathrm{Cu}(111)$ : A high resolution EELS study. Surface Science 119 (2-3), 399-410.

Gao, X., Huang, Z., Qu, J., Fang, D., 2014. A curvature-dependent interfacial energybased interface stress theory and its applications to nano-structured materials:(I) General theory. Journal of the Mechanics and Physics of Solids 66, 59-77.

Gibbs, J. W., 1928. The collected works of J. Willard Gibbs, Volume I: Thermodynamics. Longmans, Green and Company, New York, 228.

Gurtin, M. E., Murdoch, A. I., 1975. A continuum theory of elastic material surfaces. Archive for Rational Mechanics and Analysis 57 (4), 291-323.

Gurtin, M. E., Weissmüller, J., Larché, F., 1998. A general theory of curved deformable interfaces in solids at equilibrium. Philosophical Magazine A 78 (5), 1093-1109.

Hallil, A., Raulot, J.-M., Cherkaoui, M., 2014. Atomistic simulations of $\mathrm{Cu}_{2} \mathrm{O}$ bulk and $\mathrm{Cu} / \mathrm{Cu}_{2} \mathrm{O}$ interface properties by using a new interatomic potential. Computational Materials Science 81, 366-373.

Herring, C., 1951. Some theorems on the free energies of crystal surfaces. Physical Review 82 (1), 87-93.

Hu, S. Y., Chen, L.-Q., 2002. Diffuse-interface modeling of composition evolution in the presence of structural defects. Computational Materials Science 23 (1), 270282.

Jensen, F., Besenbacher, F., Lægsgaard, E., Stensgaard, I., 1991. Oxidation of $\mathrm{Cu}(111)$ : two new oxygen induced reconstructions. Surface Science 259 (3), L774L780. 
Leo, P. H., Lowengrub, J. S., Jou, H.-J., 1998. A diffuse interface model for microstructural evolution in elastically stressed solids. Acta Materialia 46 (6), 21132130.

Lipinski, P., Barhdadi, E. H., Cherkaoui, M., 2006. Micromechanical modelling of an arbitrary ellipsoidal multi-coated inclusion. Philosophical Magazine 86 (10), $1305-1326$.

Marichev, V. A., 2011. The Shuttleworth equation: Its modifications and current state. Protection of Metals and Physical Chemistry of Surfaces 47 (1), 25-30.

Mi, C., Jun, S., Kouris, D. A., Kim, S. Y., 2008. Atomistic calculations of interface elastic properties in noncoherent metallic bilayers. Physical Review B 77 (7), 075425 .

Mi, C., Kouris, D., 2012. On the significance of coherent interface effects for embedded nanoparticles. Mathematics and Mechanics of Solids (doi: 10.1177/1081286512465426).

Miller, R. E., Shenoy, V. B., 2000. Size-dependent elastic properties of nanosized structural elements. Nanotechnology 11 (3), 139.

Müller, P., Saúl, A., 2004. Elastic effects on surface physics. Surface Science Reports $54(5), 157-258$.

Murdoch, A. I., 1976. A thermodynamical theory of elastic material interfaces. The Quarterly Journal of Mechanics and Applied 29 (3), 245-275.

Murdoch, A. I., 2005. Some fundamental aspects of surface modelling. Journal of Elasticity 80 (1-3), 33-52. 
Nix, W., Gao, H., 1998. An atomistic interpretation of interface stress. Scripta Materalia 39 (12), 1653-1661.

Nozières, P., Wolf, D. E., 1988. Interfacial properties of elastically strained materials. Zeitschrift für Physik B Condensed Matter 70 (3), 399-407.

Pierson, J. F., Wiederkehr, D., Billard, A., 2005. Reactive magnetron sputtering of copper, silver, and gold. Thin Solid Films 478 (1-2), 196-205.

Povstenko, Y. Z., 1993. Theoretical investigation of phenomena caused by heterogeneous surface tension in solids. Journal of the Mechanics and Physics of Solids 41 (9), 1499-1514.

Qu, J., Bassani, J. L., 1993. Interfacial fracture mechanics for anisotropic bimaterials. Journal of Applied Mechanics 60 (2), 422-431.

Romanov, A. E., Wagner, T., 2001. On the universal misfit parameter at mismatched interfaces. Scripta Materialia 45 (3), 325-331.

Romanov, A. E., Wagner, T., Rühle, M., 1998. Coherent to incoherent transition in mismatched interfaces. Scripta Materialia 38 (6), 869-875.

Sander, D., 2003. Surface stress: implications and measurements. Current Opinion in Solid State and Materials Science 7 (1), 51-57.

Sayle, D. C., Catlow, C. R. A., Dulamita, N., Healy, M. J. F., Maicaneanu, S. A., Slater, B., Watson, G. W., 2002. Modelling oxide thin films. Molecular Simulation $28(6-7), 683-725$.

Schedel-Niedrig, T., Hävecker, M., Knop-Gericke, A., Schlögl, R., 2000. Partial 
methanol oxidation over copper: Active sites observed by means of in situ X-ray absorption spectroscopy. Physical Chemistry Chemical Physics 2 (15), 3473-3481.

Sharma, P., Ganti, S., Bhate, N., 2003. Effect of surfaces on the size-dependent elastic state of nano-inhomogeneities. Applied Physics Letters 82 (4), 535-537.

Shuttleworth, R., 1950. The surface tension of solids. Proceedings of the Physical Society. Section A: Mathematical, Physical and Engineering Sciences 63 (5), 444.

Soon, A., Todorova, M., Delley, B., Stampfl, C., 2006. Oxygen adsorption and stability of surface oxides on $\mathrm{Cu}(111)$ : A first-principles investigation. Physical Review B 73 (16), 165424.

Steigmann, D. J., Ogden, R. W., 1997. Plane deformations of elastic solids with intrinsic boundary elasticity. Proceedings of the Royal Society of London. Series A: Mathematical, Physical and Engineering Sciences 453 (1959), 853-877.

Steigmann, D. J., Ogden, R. W., 1999. Elastic surface-substrate interactions. Proceedings of the Royal Society of London. Series A: Mathematical, Physical and Engineering Sciences 455 (1982), 437-474.

Vermaak, J. S., Mays, C. W., Kuhlmann-Wilsdorf, D., 1968. On surface stress and surface tension: I. Theoretical considerations. Surface Science 12 (2), 128-133.

Werner, H., Herein, D., Schulz, G., Wild, U., Schlögl, R., 1997. Reaction pathways in methanol oxidation: kinetic oscillations in the copper/oxygen system. Catalysis Letters 49 (1-2), 109-119.

Zhang, C., Yao, Y., Chen, S., 2014. Size-dependent surface energy density of typically fcc metallic nanomaterials. Computational Materials Science 82, 372-377. 
Table 1: Geometric parameters used to set up the atomistic simulations.

\begin{tabular}{|c|c|c|c|c|c|c|}
\hline \hline Interface & $\epsilon^{m, S}(\%)$ & $n$ & $m$ & $\mathrm{~L}(\AA)$ & $\mathrm{D}(\AA)$ & $N_{\mathrm{Cu}}+N_{\mathrm{Cu}_{2} \mathrm{O}}$ \\
\hline 1 & -1.476 & 14 & 6 & 218.628 & 35.923 & 19696 \\
2 & 1.34 & 12 & 5 & 217.391 & 30.479 & 14088 \\
3 & 5.42 & 15 & 6 & 215.181 & 37.645 & 21204 \\
4 & 2.524 & 17 & 7 & 216.757 & 43.017 & 27964 \\
\hline \hline
\end{tabular}


Table 2: Interfacial elastic properties for an incoherent $\mathrm{Cu} / \mathrm{Cu}_{2} \mathrm{O}$ interface.

\begin{tabular}{|ll|c|}
\hline \hline Property & & Value \\
\hline$\Gamma_{0}$ & {$\left[\mathrm{~J} . \mathrm{m}^{-2}\right]$} & 1.102 \\
$\Gamma_{11}^{(1)}$ & {$\left[\mathrm{J} . \mathrm{m}^{-2}\right]$} & 3.00 \\
$\Gamma_{1111}^{(2)}+\Gamma_{1122}^{(2)}$ & {$\left[\mathrm{J} . \mathrm{m}^{-2}\right]$} & 2455.51 \\
$\Lambda_{33}^{(2)}$ & {$\left[\mathrm{nm} . \mathrm{GPa}^{-1}\right]$} & 0.0210 \\
$\Upsilon_{11}^{(1)}$ & {$\left[\mathrm{J} . \mathrm{m}^{-2}\right]$} & 0.213 \\
$\Upsilon_{1111}^{(2)}+\Upsilon_{1122}^{(2)}$ & {$\left[\mathrm{J} \cdot \mathrm{m}^{-2}\right]$} & 526.71 \\
$\Phi_{1111}+\Phi_{1122}$ & {$\left[\mathrm{~J} . \mathrm{m}^{-2}\right]$} & -9.24 \\
\hline \hline
\end{tabular}




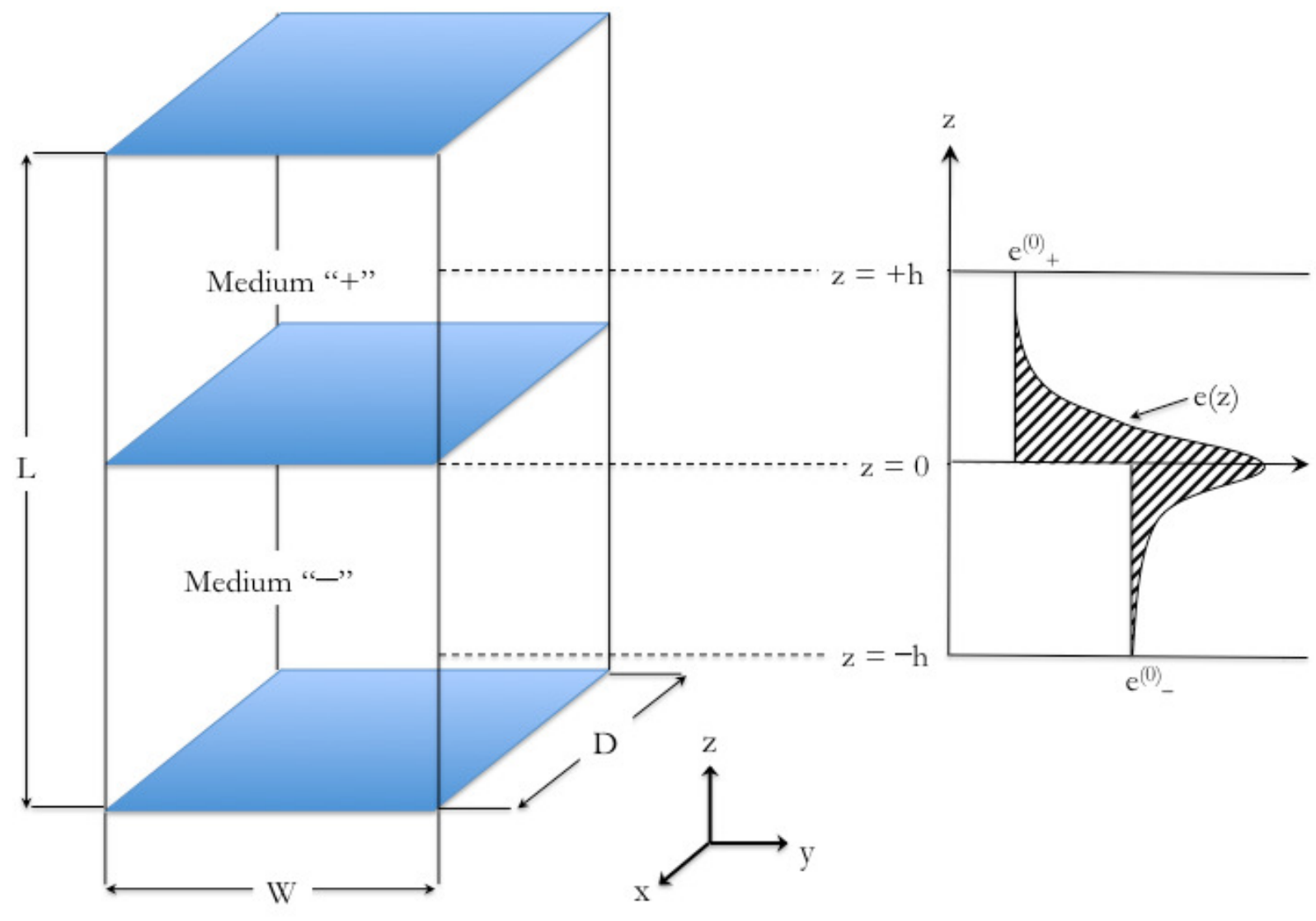

Figure 1: Schematic of a bicrystal interface and the distribution of a given excess thermodynamic quantity across the interface structure along the transverse direction $z$. 


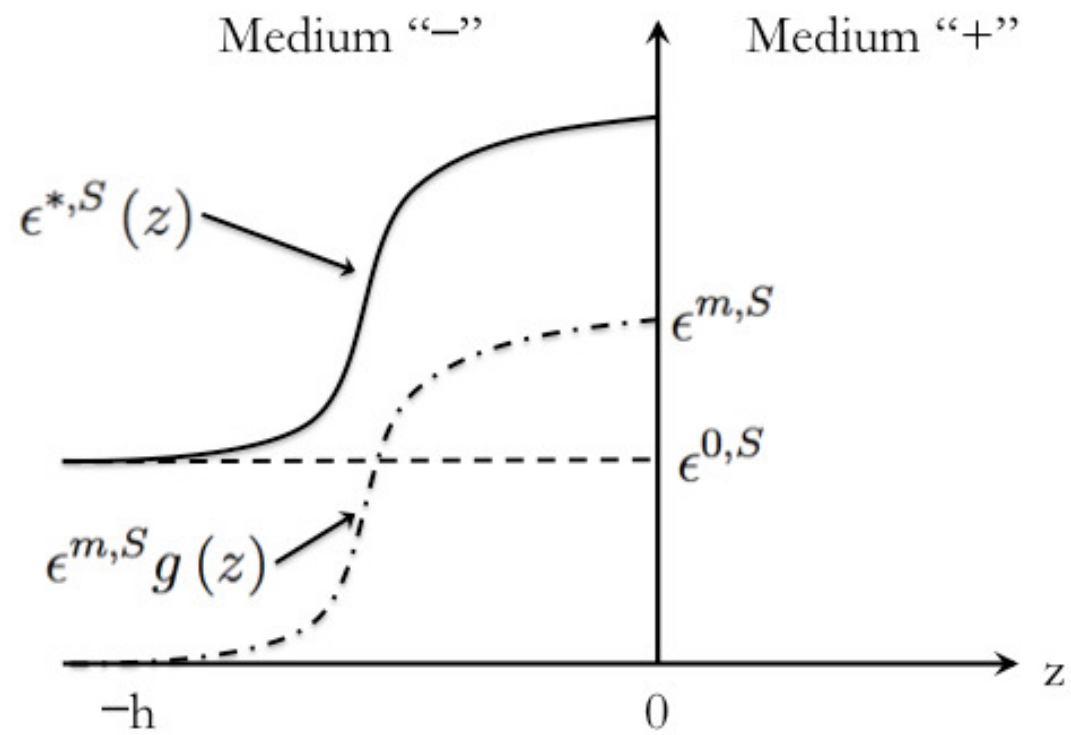

Figure 2: Decomposition of the in-plane eigenstrain $\epsilon^{*, S}$ related to the change of the interface structure. 

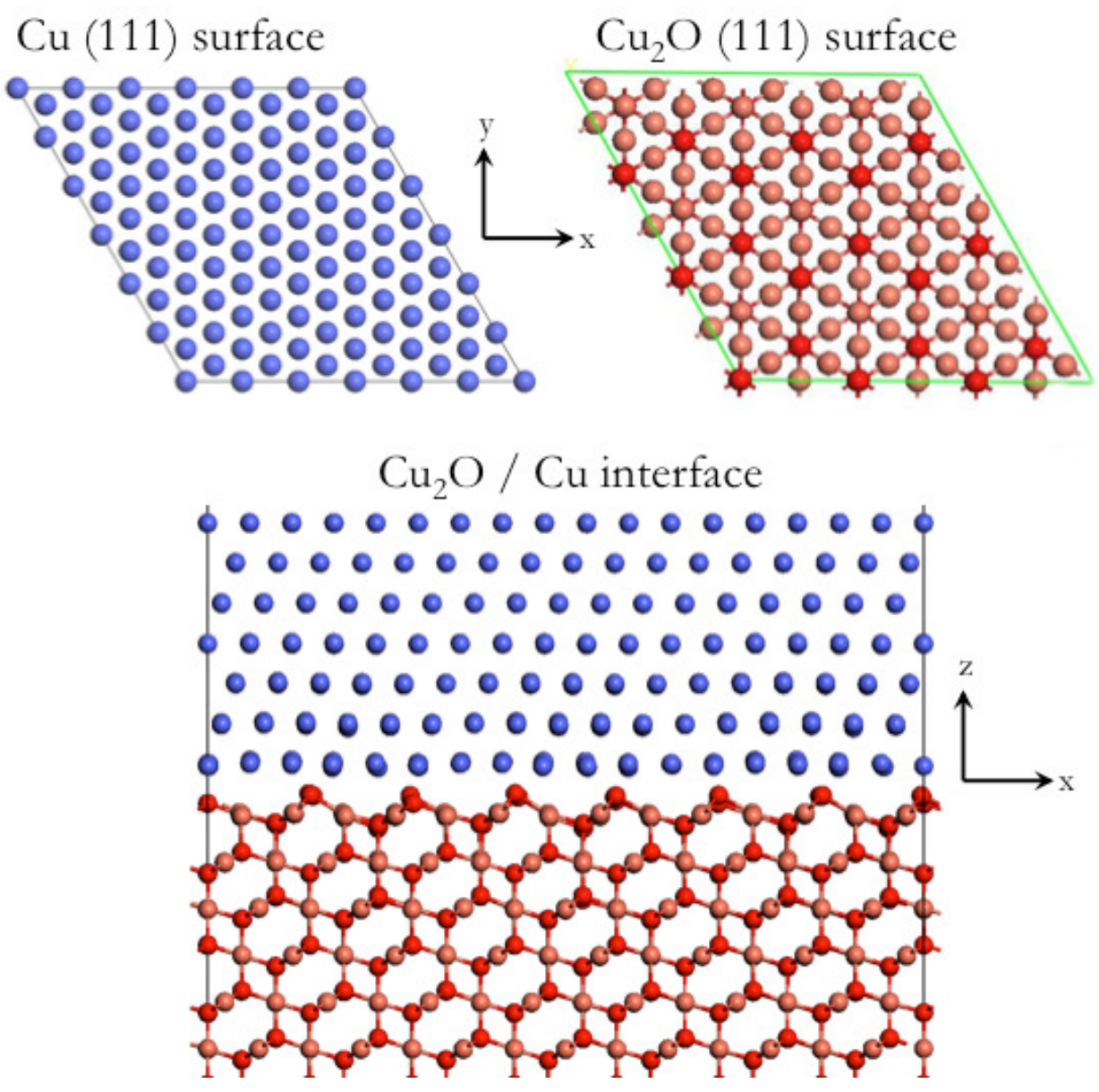

Figure 3: Illustration of the atomic surfaces composing the $\mathrm{Cu} / \mathrm{Cu}_{2} \mathrm{O}$ interface. Details of the setup of atomistic simulations are summarized in Table 1. 


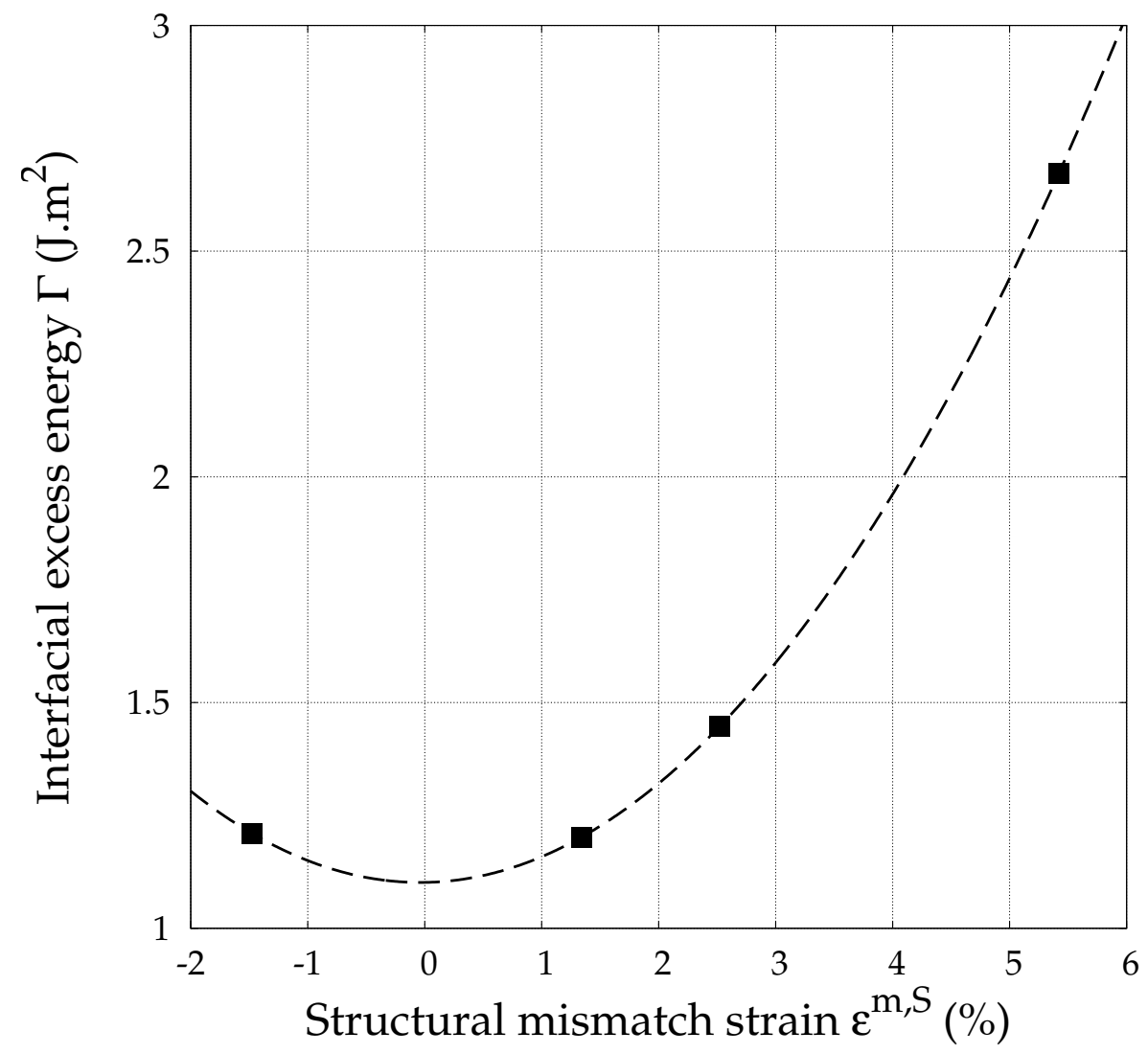

Figure 4: Variation of the interfacial excess energy $\left.\Gamma\right|_{\epsilon^{S}=0, \sigma^{\perp}=0}$ as a function of the mismatch strain $\boldsymbol{\epsilon}^{m, S}$ for an incoherent $\mathrm{Cu} / \mathrm{Cu}_{2} \mathrm{O}$ (for $\boldsymbol{\epsilon}^{S}=0$ and $\boldsymbol{\sigma}^{\perp}=0$ ). Dashed line $(---$ ) is based on the theoretical framework presented in equation (16), square symbols ( $\mathbf{\square})$ are from atomistic simulations. Interfacial elasticity properties used in equation (16) are presented in Table 2. 


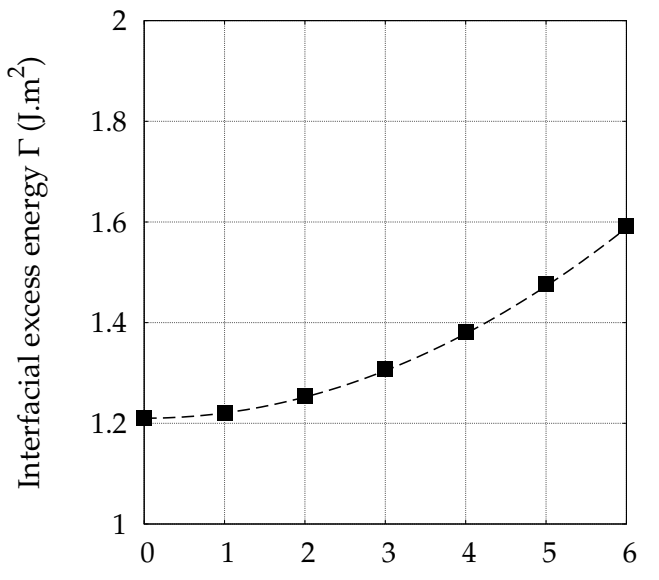

(a) Mismatch strain: $\epsilon^{m, S}=-1.476 \%$

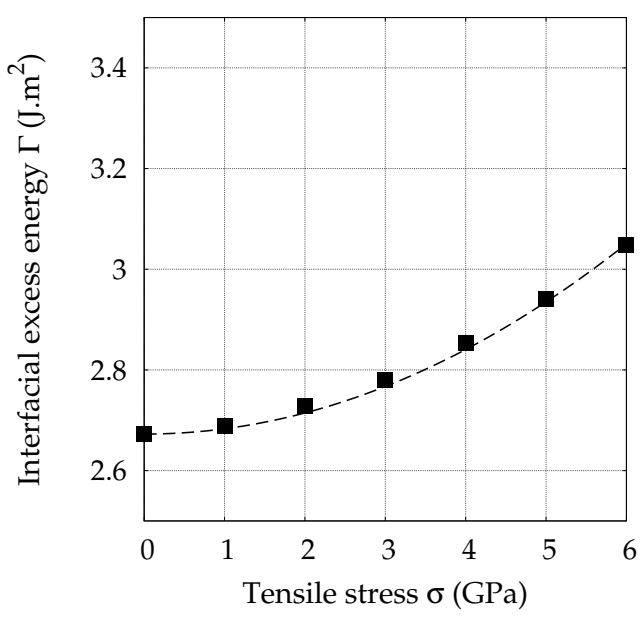

(c) Mismatch strain: $\epsilon^{m, S}=5.421 \%$

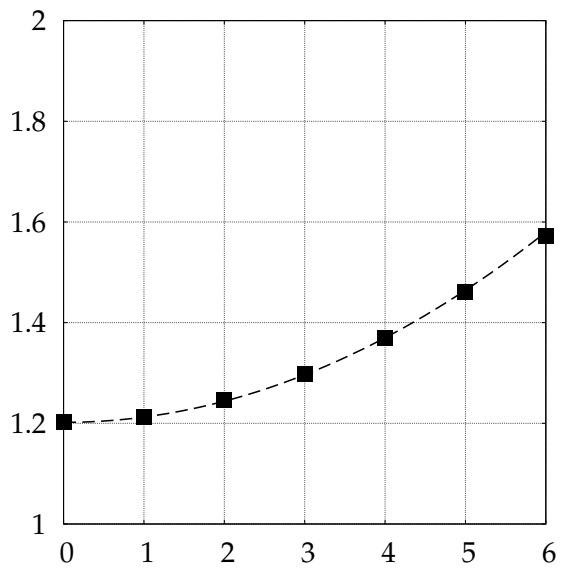

(b) Mismatch strain: $\epsilon^{m, S}=1.34 \%$

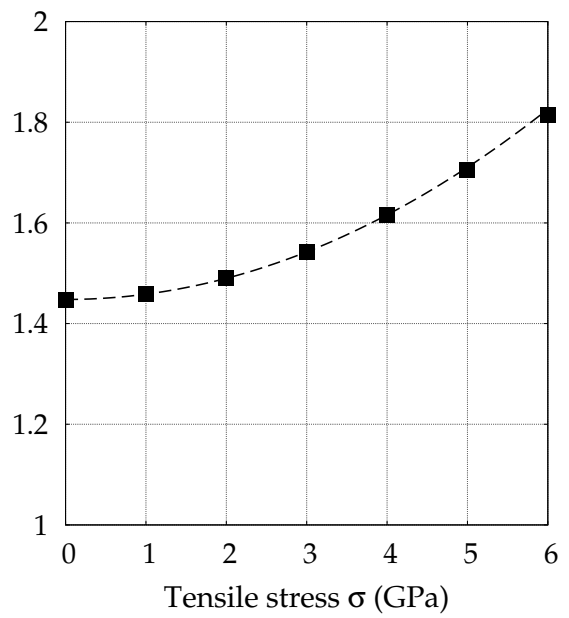

(d) Mismatch strain: $\epsilon^{m, S}=2.524 \%$

Figure 5: Variation of the interfacial excess energy $\left.\Gamma\right|_{\epsilon^{S}=0}$ as a function of the transverse tensile stress $\boldsymbol{\sigma}^{\perp}$ (for $\boldsymbol{\epsilon}^{S}=0$ ) for an incoherent $\mathrm{Cu} / \mathrm{Cu}_{2} \mathrm{O}$ interface with various mismatch strains. Dashed line $(---)$ is based on the theoretical framework presented in equation (16), square symbols are from atomistic simulations. Interfacial elasticity properties used in equation (16) are presented in Table 2. 


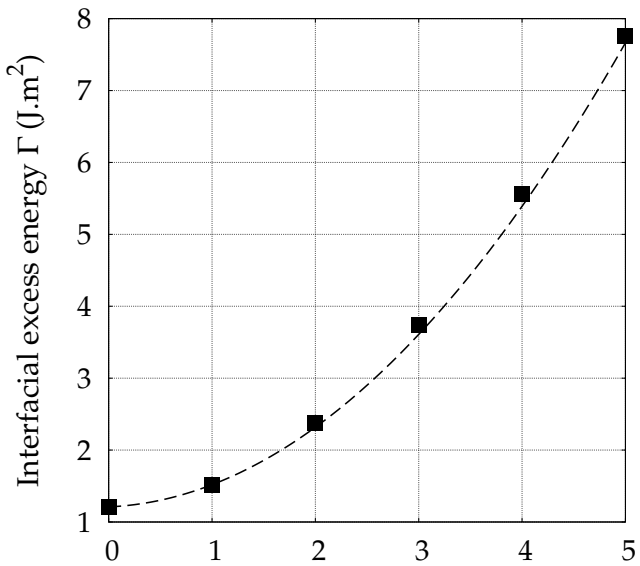

(a) Mismatch strain: $\epsilon^{m, S}=-1.476 \%$

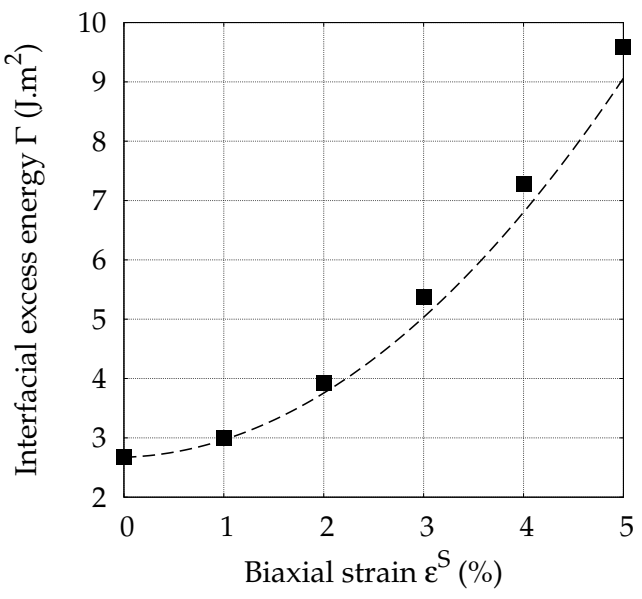

(c) Mismatch strain: $\epsilon^{m, S}=5.421 \%$

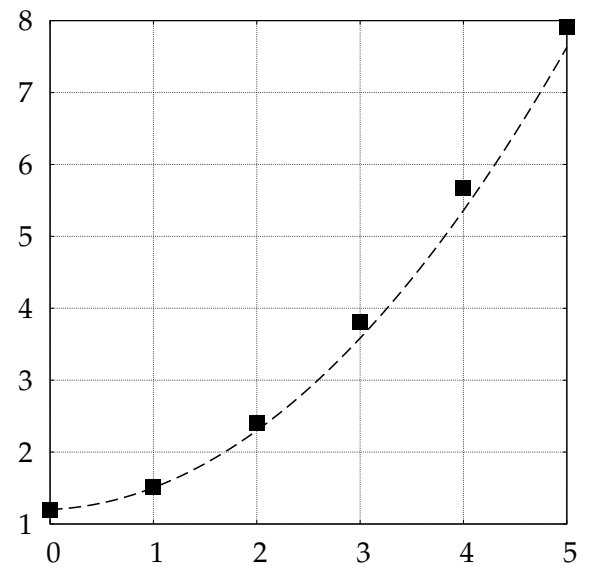

(b) Mismatch strain: $\epsilon^{m, S}=1.34 \%$

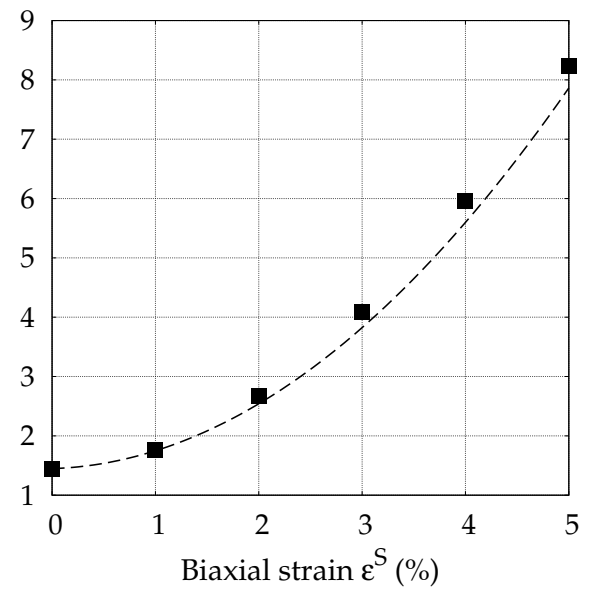

(d) Mismatch strain: $\epsilon^{m, S}=2.524 \%$

Figure 6: Variation of the interfacial excess energy $\left.\Gamma\right|_{\sigma^{\perp}=0}$ as a function of the biaxial strain $\epsilon^{S}$ (for $\boldsymbol{\sigma}^{\perp}=0$ ) for an incoherent $\mathrm{Cu} / \mathrm{Cu}_{2} \mathrm{O}$ interface with various mismatch strains. Dashed line $(---)$ is based on the theoretical framework presented in equation (16), square symbols $(\mathbf{\square})$ are from atomistic simulations. Interfacial elasticity properties used in equation (16) are presented in Table 2. 


\section{Appendix}

\section{Appendix A. Nomenclature and list of symbols}

We denote in boldface a vector a of the Euclidian space $\mathbb{E}$ only by its element $a_{i}$ (i.e. $\mathbf{a}=a_{i} \hat{\mathbf{e}}_{i}$ ) where we employ the use of Einstein's convention of summation over repeated indices. The component $\hat{\mathbf{e}}_{i}$ is an element of $\left(\hat{\mathbf{e}}_{1}, \hat{\mathbf{e}}_{2}, \hat{\mathbf{e}}_{3}\right)$, a positive oriented orthonormal basis of $\mathbb{E}$ with dimension 3. Similarly a second-rank Euclidian tensor A designated in a boldface character is simply written as $A_{i j}\left(\mathbf{A}=A_{i j} \hat{\mathbf{e}}_{i} \otimes \hat{\mathbf{e}}_{j}\right)$. A third-rank Euclidian tensor $\mathbb{A}$ designated by an underlined character is simply written as $A_{i j k}\left(\underline{\mathbb{A}}=A_{i j k} \hat{\mathbf{e}}_{i} \otimes \hat{\mathbf{e}}_{j} \otimes \hat{\mathbf{e}}_{k}\right)$. A fourth-rank Euclidian tensor $\underline{\underline{\mathbb{A}}}$ designated by a doubly underlined character is simply written as $A_{i j k l}\left(\underline{\underline{\mathbb{A}}}=A_{i j k l} \hat{\mathbf{e}}_{i} \otimes \hat{\mathbf{e}}_{j} \otimes \hat{\mathbf{e}}_{k} \otimes \hat{\mathbf{e}}_{l}\right)$. A character not in boldface or not underlined indicates a scalar. The symbol "." between two tensors designates the product contracted once, while the symbol ":" designates the product contracted twice. Roman indices used in tensorial notations range from 1 to 3 (three-dimensional) and Greek indices range from 1 to 2 (twodimensional), unless otherwise indicated.

\section{Notation indexing:}

$[\cdot]_{ \pm} \quad-$ denotes a variable defined in medium "+" or "_" respectively.

$[\cdot]^{S} \quad-$ denotes a variable defined in the plane of the interface.

$[\cdot]^{\perp} \quad-$ denotes a variable defined transverse to the interface.

\section{Strain and stress definitions:}

$\boldsymbol{\epsilon}^{S} \quad$ - interfacial in-plane strain tensor.

$\epsilon^{*, S} \quad-$ interfacial structure eigenstrain tensor. 


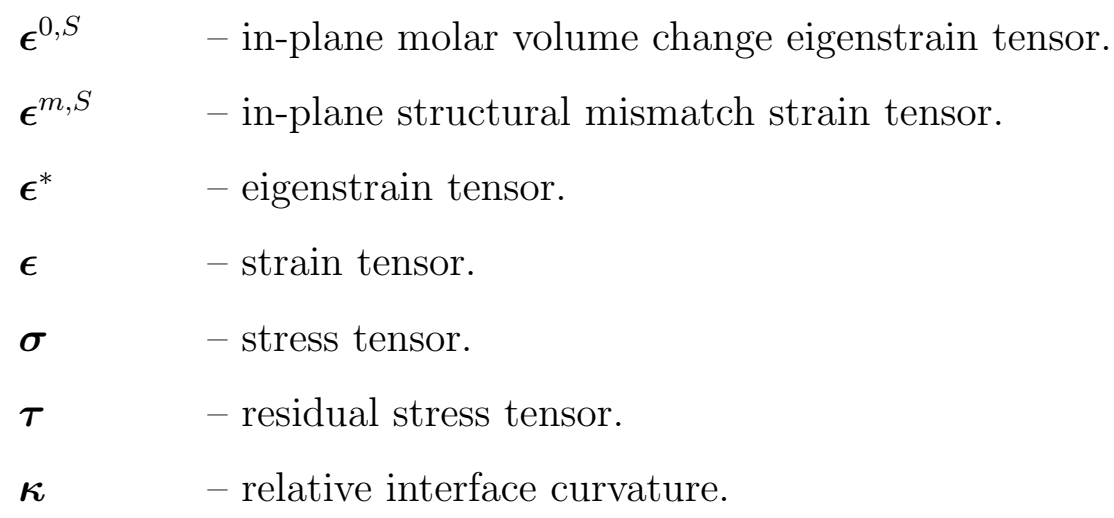

$\mathcal{F} \quad-$ total free energy.

$\Psi \quad$ - bulk free-energy density per unit volume.

$p^{S} \quad$ - interfacial power density of internal forces.

$\Gamma \quad-$ interfacial excess energy.

$\Gamma_{0} \quad-$ residual interfacial excess energy.

$\Sigma^{S} \quad-$ coherent surface stress.

$\Upsilon^{S} \quad$ - incoherent surface stress.

$\Delta^{\perp} \quad-$ transverse excess strain.

$\mathbb{D}^{\perp} \quad$ - thermodynamic conjugate of the transverse stress field $\boldsymbol{\sigma}^{\perp}$.

$\Gamma^{(1)} \quad-$ residual surface stress.

$\Lambda^{(1)} \quad-$ residual transverse interfacial deformation.

$\boldsymbol{\Upsilon}^{(1)} \quad-$ residual incoherent surface stress.

$M^{S} \quad$ - interface bending moment. 


$\begin{array}{ll}\lambda & - \text { Lamé constant. } \\ \mu & - \text { shear modulus. } \\ \alpha & - \text { coefficient of thermal expansion. } \\ \underline{\underline{\mathbb{C}}} & - \text { stiffness tensor. } \\ \mathbb{M} & - \text { compliance tensor. } \\ \underline{\gamma} & - \text { transverse coupling tensor. }\end{array}$

\section{Interfacial constants:}

$\underline{\underline{\Gamma}}^{(2)} \quad$ - interface in-plane elastic stiffness tensor.

$\Lambda^{(2)} \quad$ - interface transverse compliance tensor.

$\underline{\underline{\Upsilon}}^{(2)} \quad$ - interface in-plane incoherency stiffness tensor.

$\underline{\underline{\Phi}} \quad-$ interface in-plane structural interfacial mismatch coupling tensor.

$\underline{\mathbb{H}} \quad-$ interfacial Poisson's effect tensor.

$\underline{\mathbb{K}} \quad$ - interface transverse structural interfacial mismatch tensor.

$\lambda^{S}, \lambda^{*, S} \quad$ - surface Lamé constants.

$K^{S}, K^{*, S}$ - biaxial surface moduli.

$\nu^{S}, \nu^{*, S} \quad$ - interfacial Poisson's ratios.

$E^{\perp}, E^{*, \perp}$ - interfacial transverse Young's moduli.

$\alpha^{S} \quad-$ interfacial coefficient of thermal expansion.

$d_{0}, d_{0}^{*} \quad$ - thermal-mechanical coupling coefficient.

\section{Variables used in the atomistic model:}

$e^{(i)} \quad-$ total energy of atom $i$.

$e^{(0)} \quad-$ atomic bulk energy.

$N \quad-$ number of atoms.

$n, m \quad-$ scaling factors to obtain various mismatch strains in the atomistic model. 
$\ell \quad-$ crystal lattice parameter.

$W, L, D \quad$ - dimensions of the atomistic simulation box.

$A_{0} \quad$ - surface area.

840

$\mathcal{S} \quad-$ imaginary, two-dimensional surface.

$\Omega \quad-$ semi-infinite crystal.

z $\quad-$ position across the interface.

$h-$ arbitrary distance away from the interface.

X $\quad-$ position vector.

$\mathbf{x}^{S} \quad-$ interfacial position vector.

u $\quad-$ displacement field.

$\nabla^{S} \quad-$ surface gradient.

$\mathbf{n}\left(\mathbf{x}^{S}\right) \quad$ - interface unit normal upward pointing from the reference phase.

$V^{0} \quad-$ molar volume.

$g(\mathbf{x}) \quad$ - variation of the structural mismatch.

\section{Appendix B. "T-decomposition": General anisotropic elasticity with eigen-} strains due to lattice mismatch

In the following "T-decomposition" (Qu and Bassani, 1993; Dingreville and Qu, 2008), subscripts $\alpha, \beta, \kappa, \lambda$ vary from 1 to 2 , and Roman subscripts $j$ vary from 1 to 3 with the following conventions:

$$
\begin{gathered}
\sigma_{\alpha \beta}^{S}=\sigma_{\alpha \beta}, \quad \tau_{\alpha \beta}^{S}=\tau_{\alpha \beta}, \quad \tau_{\alpha \beta}^{S}=\tau_{\alpha \beta}, \quad \epsilon_{\kappa \lambda}^{S}=\epsilon_{\kappa \lambda}, \\
\sigma_{j}^{\perp}=\sigma_{3 j}, \quad \tau_{j}^{\perp}=\tau_{3 j}, \quad \epsilon_{\alpha}^{\perp}=2 \epsilon_{\alpha 3}, \quad \epsilon_{3}^{\perp}=\epsilon_{33},
\end{gathered}
$$


where $\mathbb{M}_{k j}^{\perp}$ and $\gamma_{k \alpha \beta}$ are given by:

$$
\mathbb{M}_{k j}^{\perp}=\mathbb{C}_{3 k 3 j}^{-1} \quad, \quad \gamma_{k \alpha \beta}=\mathbb{M}_{k j}^{\perp} \mathbb{C}_{3 j \alpha \beta}
$$


${ }_{859}$ Introducing equation (B.7) into equation (B.5), the surface stress tensor $\sigma_{\alpha \beta}^{S}$ is given 860 by:

$$
\sigma_{\alpha \beta}^{S}=\hat{\tau}_{\alpha \beta}^{S}+\mathbb{C}_{\alpha \beta \kappa \lambda}^{S}\left(\epsilon_{\kappa \lambda}^{S}-\epsilon_{\kappa \lambda}^{*, S}\right)+\gamma_{j \alpha \beta} \sigma_{j}^{\perp}
$$

861 where

$$
\hat{\tau}_{\alpha \beta}^{S}=\tau_{\alpha \beta}^{S}-\tau_{j}^{\perp} \gamma_{j \alpha \beta} \quad, \quad \mathbb{C}_{\alpha \beta \kappa \lambda}^{S}=\mathbb{C}_{\alpha \beta \kappa \lambda}-\mathbb{C}_{\alpha \beta 3 k} \gamma_{k \kappa \lambda}
$$

862 Introducing both equations (B.7) and (B.9) into equation (B.4), the energy density 86з $\Psi$ finally becomes:

$$
\Psi=\hat{\Psi}_{0}+\hat{\tau}_{\alpha \beta}^{S}\left(\epsilon_{\alpha \beta}^{S}-\epsilon_{\alpha \beta}^{*, S}\right)+\frac{1}{2} \mathbb{C}_{\alpha \beta \kappa \lambda}^{S}\left(\epsilon_{\alpha \beta}^{S}-\epsilon_{\alpha \beta}^{*, S}\right)\left(\epsilon_{\kappa \lambda}^{S}-\epsilon_{\kappa \lambda}^{*, S}\right)+\frac{1}{2} \sigma_{j}^{\perp} \mathbb{M}_{j k}^{\perp} \sigma_{k}^{\perp}
$$

864 where

$$
\hat{\Psi}_{0}=\Psi_{0}-\frac{1}{2} \mathbb{M}_{j k} \tau_{j}^{\perp} \tau_{k}^{\perp}
$$

